\title{
SECOND-ORDER SUFFICIENT OPTIMALITY CONDITIONS FOR THE OPTIMAL CONTROL OF NAVIER-STOKES EQUATIONS*
}

\author{
Fredi Tröltzsch ${ }^{1}$ And Daniel WaChSmuth ${ }^{1}$
}

\begin{abstract}
In this paper sufficient optimality conditions are established for optimal control of both steady-state and instationary Navier-Stokes equations. The second-order condition requires coercivity of the Lagrange function on a suitable subspace together with first-order necessary conditions. It ensures local optimality of a reference function in a $L^{s}$-neighborhood, whereby the underlying analysis allows to use weaker norms than $L^{\infty}$.
\end{abstract}

Mathematics Subject Classification. 49K20, 49K27.

Received April 30, 2004. Revised December 13, 2004.

\section{INTRODUCTION}

In this paper, we discuss second-order sufficient optimality conditions for optimal control problems governed by steady-state and instationary Navier-Stokes equations. These conditions form a central issue for different mathematical questions of optimal control theory. If second-order sufficient conditions hold true at a given control satisfying the first-order necessary conditions, then this control is locally optimal, it is unique as a local solution, and it is stable with respect to certain perturbations of given data. Moreover, the convergence of numerical approximations (say by finite elements) can be proven, and numerical algorithms such as SQP methods can be shown to locally converge. These remarks show that second-order sufficient conditions are indispensible for a complete numerical analysis of nonconvex optimal control problems.

Consequently, second-order conditions have been important assumptions in many papers on optimal control theory of ordinary differential equations, and they became important for partial differential equations as well. We only mention the case of elliptic equations studied by Bonnans [4], Casas, Unger, and Tröltzsch [10], Casas and Mateos [9], the discussion of pointwise state-constraints in Casas, Unger, and Tröltzsch [11], or the convergence analysis of SQP methods in Arada, Raymond and Tröltzsch [3]. The papers mentioned above are concerned with semilinear elliptic and parabolic equations with nonlinearities given by Nemytski operators. Therefore, the associated state functions have to be continuous to make these operators twice continuously differentiable.

The situation is, in some sense, easier for the Navier-Stokes equations. The nonlinearity $(y \cdot \nabla) y$ appearing in these equations is of quadratic type, and the associated Taylor expansion terminates after the second-order term with zero remainder. This property has been addressed by Desai and Ito [14] and Hinze [21] for the optimal

\footnotetext{
Keywords and phrases. Optimal control, Navier-Stokes equations, control constraints, second-order optimality conditions, firstorder necessary conditions.

* This work was supported by SFB 557 "Control of complex turbulent shear flows", Technical University of Berlin.

1 Institut für Mathematik, Technische Universität Berlin, Str. d. 17. Juni 136, 10632 Berlin, Germany;

troeltz@math.tu-berlin.de; wachsmut@math.tu-berlin.de
}

(c) EDP Sciences, SMAI 2006 
control of stationary and instationary Navier-Stokes equations. It simplifies the application of second-order conditions, since spaces of $L^{2}$-type for the control and $W(0, T)$-type for the state function are appropriate.

In [26], it was shown for the case of steady-state Navier-Stokes equations that second-order conditions are sufficient for Lipschitz stability of optimal solutions with respect to perturbations. However, second-order conditions were applied in a quite strong form without showing their sufficiency for local optimality.

Here, the issue of second-order sufficiency is studied more detailed. We present the conditions in a fairly weak form that invokes also first-order sufficient conditions. More precisely, by using strongly active control constraints we shrink the subspace where the second derivative of the Lagrange function must be positive definite. Moreover, we carefully study the norms underlying the neighborhood, where local optimality can be assured, which enables us to prove local optimality in an $L^{s}$-neighborhood of the reference control with $s<\infty$. We discuss the steady-state and instationary Navier-Stokes equations in one paper, since the arguments are very similar for both cases.

If box-constraints are imposed on the controls, then strongly active control-constraints should be considered in the second-order sufficient conditions. There are simple finite-dimensional optimization problems with boxconstraints, where the Hessian matrix is negative definite, but the sufficient conditions are still satisfied. The reason for this are strongly active constraints. It is therefore clear that they should be considered also in optimal control, whenever box-constraints are given.

As strongly active constraints are concerned, we follow an approach by Dontchev, Hager, Poore and Yang [15] that has been successfully applied in other papers on second-order conditions as well. A characteristic element in the analysis of second-order conditions is the interplay between two norms, usually $L^{2}$ - and $L^{\infty}$-norms. This is necessary to deal with the well-known two-norm discrepancy that appears in the discussion of active constraints. At this point the method of proof differs from that presented by Maurer and Zowe [23], who did not address this difficulty. By this technique, a certain gap between second-order necessary and second-order sufficient conditions appears. This gap seems to be natural for problems in infinite-dimensional spaces. In a paper by Bonnans and Zidani [5], the gap was tightened for elliptic problems under the assumption that the second-order derivative of the Lagrangian defines a Legendre form. Casas and Mateos [8] extended the applicability of this concept by an assumption of positivity on the second derivative of the Hamiltonian with respect to the control. Using these techniques, we also resolve the problem of the two-norm discrepancy: an appropriate formulation of the sufficient optimality condition implies $L^{2}$-quadratic growth of the objective in a $L^{2}$-neighborhood of the reference control.

Our arguments are influenced by various papers, where first-order necessary optimality conditions and numerical methods for optimal control of instationary Navier-Stokes equations are presented. We only mention Abergel and Temam [1], Casas [7], Desai and Ito [14], Gunzburger [18], Gunzburger and Manservisi [20], Fattorini and Sritharan [17], Hinze [21], Hinze and Kunisch [22], Sritharan [27] and the reference cited therein. We partially repeat some known arguments for proving first-order necessary conditions only for convenience.

In view of the importance of second-order sufficient conditions, their numerical verification is a natural desire. Unfortunately, this is a delicate question that has not yet been solved satisfactorily till now. This concerns optimal control problems for ODEs as well as PDEs: Any numerical check is connected with some discretization so that second-order conditions are verified in some finite-dimensional setting.

For instance, the eigenvalues of the reduced Hessian matrix can be computed for the finite-dimensional model to verify its definiteness, see Mittelmann and Tröltzsch [24]. However, it seems to be impossible to deduce from the numerical result the coercivity for the infinite-dimensional setting. In the control of Navier-Stokes equations, it is known for tracking type functionals that small residuals are sufficient for second-order conditions to hold, cf. Hinze and Kunisch [22]. Here, the problem is open whether the optimal residual is really small enough in function space. The numerical method determines the discrete solution. If its associated remainder is small, the same should hold for the continuous solution - provided that it is close to the discrete one. But this can only be expected, if second-order conditions are satisfied for the solution of the infinite-dimensional problem. 
In the case of ODEs, the solvability of certain Riccati-equations can be investigated to check second-order conditions. Again, the numerical implementation of this concept needs discretization. It is not clear if the result contains reliable information on the continuous case.

Eigenvalues of the reduced Hessian, small remainders, and in the case of ODEs, the solution of Riccati equations, are useful numerical techniques to check second-order conditions. However, their numerical results will never give complete evidence for the original problem, since the computed discrete solution will be close to the exact one only under the assumption of a second-order sufficient condition. In this way, we encounter a circular reasoning.

In view of these remarks, one should handle second-order sufficient conditions similarly as constraintqualifications in nonlinear programming: they cannot be checked in general, but they should be assumed to perform a satisfactory analysis.

\section{The optimal CONTROL PROBlems}

\subsection{Control of the steady-state Navier-Stokes equations}

In the first part of the paper, we consider the optimal control problem to minimize

$$
J(u, y)=\int_{\Omega}\left|y(x)-y_{d}(x)\right|^{2} \mathrm{~d} x+\frac{\gamma}{2} \int_{\Omega}|u(x)|^{2} \mathrm{~d} x
$$

subject to the steady-state Navier-Stokes equations,

$$
\begin{array}{rlrl}
-\nu \Delta y+(y \cdot \nabla) y+\nabla p & =u & & \text { in } \Omega, \\
\operatorname{div} y=0 & & \text { in } \Omega, \\
y & =0 & & \text { on } \Gamma,
\end{array}
$$

and the box constraints

$$
u_{a}(x) \leq u(x) \leq u_{b}(x)
$$

to be fulfilled a.e. on $\Omega$. In this setting $\Omega$ is an open bounded Lipschitz domain in $\mathbb{R}^{n}$ with boundary $\Gamma$. In the steady-state case, we will restrict the space dimension $n$ to $2 \leq n \leq 4$. In this case, $H_{0}^{1}(\Omega)$ is continuously imbedded in $L^{4}(\Omega)$.

To complete the problem setting, we require the desired function $y_{d}$ to be an element of $L^{2}(\Omega)^{n}$. The parameters $\gamma$ and $\nu$ are assumed to be positive constants. In the box constraints on $u$ two functions $u_{a}, u_{b} \in$ $L^{s}(\Omega)^{n}$ are given, satisfying $u_{a, i}(x) \leq u_{b, i}(x)$ for all $i=1 \ldots n$ and almost all $x \in \Omega$. The exponent $s$ will be precised later. We set

$$
U_{a d}=\left\{u \in L^{s}(\Omega)^{n}: u_{a, i}(x) \leq u_{i}(x) \leq u_{b, i}(x), i=1 \ldots n, \text { a.e. on } \Omega\right\}
$$

Up to now we did not explain, in which sense the state equations (2.2) has to be solved. The state $y$ associated with $u$ is defined as a weak solution of (2.2) in the next section.

\subsection{The instationary case}

In the second part, we consider the optimal control problem to minimize

$$
\begin{aligned}
J(u, y)=\frac{1}{2} & \int_{\Omega}\left|y(x, T)-y_{T}(x)\right|^{2} \mathrm{~d} x+\frac{1}{2} \int_{0}^{T} \int_{\Omega}\left|y(x, t)-y_{Q}(x, t)\right|^{2} \mathrm{~d} x \mathrm{~d} t \\
& +\frac{\gamma}{2} \int_{0}^{T} \int_{\Omega}|u(x, t)|^{2} \mathrm{~d} x \mathrm{~d} t
\end{aligned}
$$


subject to the instationary Navier-Stokes equations.

$$
\begin{aligned}
y_{t}-\nu \Delta y+(y \cdot \nabla) y+\nabla p & =u & & \text { in } Q, \\
\operatorname{div} y & =0 & & \text { in } Q, \\
y(0) & =y_{0} & & \text { in } \Omega,
\end{aligned}
$$

and the control constraints $u \in U_{a d}$ with control set re-defined below, where $Q=(0, T) \times \Omega$. Here, functions $y_{T} \in L^{2}(\Omega)^{n}, y_{Q} \in L^{2}(Q)^{n}$, and $y_{0} \in H \subset L^{2}(\Omega)^{n}$ are given. The parameters $\gamma$ and $\nu$ are adopted from the last section. Let two functions $u_{a}, u_{b} \in L^{s}(Q)^{n}$ be given such that $u_{a, i}(x, t) \leq u_{b, i}(x, t)$ holds almost everywhere on $Q$ and for all $i=1, \ldots, n$. The set of admissible controls is now defined by

$$
U_{a d}=\left\{u \in L^{s}(Q)^{n}: u_{a, i}(x, t) \leq u_{i}(x, t) \leq u_{b, i}(x, t) \text { a.e. on } Q\right\} .
$$

Again, the exponent $s$ will be specified later.

\section{Optimality CONDitions FOR the STEADY-STATE PROBlem}

In this section, we provide basic results on the state equation and first-order necessary optimality conditions. These results are more or less known from the literature. However, they are mostly presented in a different form and not directly applicable for our purposes. Therefore, we recall them for convenience.

\subsection{The state equation}

First, we define a solenoidal space that is frequently used in the literature,

$$
V:=\left\{v \in H_{0}^{1}(\Omega)^{n}: \operatorname{div} v=0\right\} .
$$

This space is a Hilbert space endowed with the standard scalar product of $H_{0}^{1}$,

$$
(y, v)_{V}=\sum_{i=1}^{n}\left(\nabla y_{i}, \nabla v_{i}\right)_{L^{2}(\Omega)}
$$

The associated norm is denoted by $|\cdot|_{V}$. Further on, we will denote the pairing between $V^{\prime}$ and $V$ as $\langle f, v\rangle$, where $f \in V^{\prime}$ and $v \in V$. To simplify the notation, we define for $u \in L^{q}(\Omega)^{n}$

$$
|u|_{q}:=|u|_{L^{q}(\Omega)^{n}} .
$$

The pairing between $L^{q}(\Omega)^{n}$ and $L^{q^{\prime}}(\Omega)^{n}$ is denoted by $(\cdot, \cdot)_{q, q^{\prime}}, 1 / q+1 / q^{\prime}=1$. For $q=q^{\prime}=2$ we get the usual scalar product of $L^{2}(\Omega)^{n}$, and we write $(\cdot, \cdot)_{2}:=(\cdot, \cdot)_{2,2}$. In the following, we will make use of the well-known interpolation inequality, $c f$. Brezis [6].

Lemma 3.1. Let $1 \leq q \leq 2$ be given. Define $s$ by $s=q /(2-q)$ for $q<2$, or $s=\infty$ for $q=2$, respectively. Further, let $D \subset \mathbb{R}^{m}$ be a bounded and measurable set. Then it holds for all $u \in L^{s}(D)$

$$
|u|_{L^{q}(D)}^{2} \leq|u|_{L^{1}(D)}|u|_{L^{s}(D)} .
$$

Additionally, we need the following well-known lemma of imbeddings of $L^{p}$-spaces, $c f$. Adams [2].

Lemma 3.2. Let $D \subset \mathbb{R}^{m}$ be a bounded and measurable set with $\operatorname{vol}(D):=\int_{D} 1 \mathrm{~d} x<\infty$, and let $1 \leq p \leq q \leq \infty$ be given. Then for all $u \in L^{q}(D)$ it holds

$$
|u|_{L^{p}(D)} \leq(\operatorname{vol}(D))^{1 / p-1 / q}|u|_{L^{q}(D)} .
$$


Let us introduce for convenience a trilinear form $b: V \times V \times V \mapsto \mathbb{R}$ by

$$
b(u, v, w)=((u \cdot \nabla) v, w)_{2}=\int_{\Omega} \sum_{i, j=1}^{n} u_{i} \frac{\partial v_{j}}{\partial x_{i}} w_{j} \mathrm{~d} x
$$

The following result was proven in [28].

Lemma 3.3. For all $u, v, w \in V$ it holds

$$
b(u, v, w)=-b(u, w, v) .
$$

There is a positive constant $C_{n}$ depending on the dimension $n$ but not on $u, v, w$ and $\Omega$, such that

$$
|b(u, v, w)| \leq C_{n}|u|_{4}|v|_{V}|w|_{4}
$$

holds for all $u, v, w \in V$.

As a simple conclusion of the previous lemma, we get $b(u, v, v)=0$ for all $u, v \in V$. The estimate (3.1) expresses the continuity of $b$. We refer to $[12,21]$ for further estimates of $b$.

Furthermore, we introduce for $p \leq 2 n /(n-2)$ by $N_{p}$ the norm of the imbedding of $H_{0}^{1}(\Omega)^{n}$ in $L^{p}(\Omega)^{n}$, i.e. $|y|_{p} \leq N_{p}|y|_{H_{0}^{1}(\Omega)^{n}}$. For $2 \leq n \leq 4$, the imbedding of $H_{0}^{1}(\Omega)^{n}$ in $L^{4}(\Omega)^{n}$ is continuous. This fact will be frequently used. Moreover, we conclude from (3.1)

$$
|b(u, v, w)| \leq C_{n} N_{4}^{2}|u|_{V}|v|_{V}|w|_{V} \quad \forall u, v, w \in V
$$

To obtain optimal regularity properties of the control-to-state mapping, we select real numbers $q, q^{\prime}, s$ satisfying the following assumption

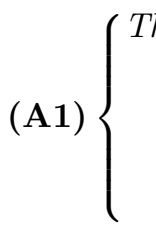
The numbers $q, q^{\prime}, s \geq 1$ satisfy the following conditions:
(i) The imbedding of $H_{0}^{1}(\Omega)$ in $L^{q^{\prime}}(\Omega)$ is continuous.
(ii) The exponents $q$ and $q^{\prime}$ are conjugate exponents, i.e. $1 / q+1 / q^{\prime}=1$.
(iii) For all $u \in L^{s}(\Omega)^{n}$ it holds$$
|u|_{q}^{2} \leq|u|_{1}|u|_{s}
$$

Notice that condition (iii) implies $q \leq 2$. Here we have in mind two different situations. At first, $q=q^{\prime}=2$ and $s=\infty$ meet this assumption. Then the second-order sufficient condition of section 3.3 yields local optimality of the reference control in a $L^{s}=L^{\infty}$-neighbourhood. This means more or less that jumps of the optimal control have to be known a-priorily. To overcome this difficulty, we employ a second configuration, namely $q^{\prime}=4, q=4 / 3, s=2$, confer Lemma 3.1. Here we are able to work with a $L^{2}$-neighbourhood of the reference control.

The use of the $L^{s}$-neighbourhoods with $s<\infty$ is possible since the control $u$ appears linearly in the equation and quadratically in the objective. Moreover, control and state are separated in the objective funtional.

Definition 3.4 (weak solution). Let $u \in L^{q}(\Omega)^{n}$ be given. A function $y \in V$ is called weak solution of (2.2) if it satisfies the variational equation

$$
\nu(y, v)_{V}+b(y, y, v)=(u, v)_{q, q^{\prime}} \quad \forall v \in V
$$


Observe that $v \in V$ implies $v \in L^{q^{\prime}}(\Omega)$. This fact permits us to work with controls that are less regular than $L^{2}(\Omega)^{n}$. Moreover, we recall $(-\nu \Delta y, v)_{2}=\nu(y, v)_{V}$. It is known that (3.3) admits a unique solution $y$ if the norm of the inhomogeneity $u$ is sufficiently small or the coefficient $\nu$ is sufficiently large:

Theorem 3.5 (existence and uniqueness of weak solutions). For given $f \in V^{\prime}$ the equation

$$
\nu(y, v)_{V}+b(y, y, v)=\langle f, v\rangle \quad \forall v \in V
$$

admits at least one solution $y \in V$. If the smallness condition

$$
\nu^{2}>C_{n} N_{4}^{2}|f|_{V^{\prime}}
$$

is satisfied, then this solution is unique.

This is proven for instance in [28], Theorems II.1.2, II.1.3.

If the functional $f$ in (3.4) is generated by a $L^{q}$-function $u$,

$$
\langle f, v\rangle=(u, v)_{q, q^{\prime}},
$$

then we have to impose some restrictions on the $L^{q}(\Omega)$-norm of $u$. Let the $L^{q}(\Omega)$-norm of the admissible controls be bounded by $\mathcal{M}_{q}$, i.e.

$$
\mathcal{M}_{q}=\sup _{u \in U_{a d}}|u|_{q}
$$

Then the following condition ensures existence and uniqueness of $y=y(u)$ :

$$
\text { (A2) }\left\{\begin{array}{l}
\text { The set of admissible controls } U_{a d} \text { is bounded in } L^{q}(\Omega)^{n} \text {. The bound } \mathcal{M}_{q} \text { satifies, together with the } \\
\text { viscosity parameter } \nu, \\
\frac{2 C_{n} N_{q^{\prime}} N_{4}^{2}}{\nu^{2}} \mathcal{M}_{q} \leq 1 .
\end{array}\right.
$$

In the sections dealing with the steady-state case we assume that these two Assumptions (A1) and (A2) are satisfied. The solution mapping $u \mapsto y$ can be estimated directly by standard methods, see for instance [28].

Lemma 3.6. For all $u \in U_{a d}$, the variational equality (3.3) admits a unique solution $y \in V$. If $y_{1}, y_{2} \in V$ are weak solutions of (3.3) corresponding to $u_{1}, u_{2} \in U_{a d}$, then

$$
\left|y_{i}\right|_{V} \leq \frac{N_{q^{\prime}}}{\nu}\left|u_{i}\right|_{q} \leq \frac{N_{q^{\prime}}}{\nu} \mathcal{M}_{q} \text { and }\left|y_{1}-y_{2}\right|_{V} \leq \frac{2 N_{q^{\prime}}}{\nu}\left|u_{1}-u_{2}\right|_{q}
$$

i.e. the solution mapping $u \mapsto y$ is Lipschitz on $U_{a d}$.

Proof. Existence and uniqueness of solutions follow by Theorem 3.5 in view of assumption (3.5). Testing (3.3) with $v=y$ yields

$$
\nu|y|_{V}^{2}+b(y, y, y)=(u, y)_{q, q^{\prime}} \leq|u|_{q}|y|_{q^{\prime}} \leq \frac{\nu}{2}|y|_{V}^{2}+\frac{N_{q^{\prime}}^{2}}{2 \nu}|u|_{q}^{2}
$$

by the Young inequality. Since $b(y, y, y)=0$ for all $y \in V$, the first estimate follows immediately. The second is obtained in the following way: We test the variational equalities for $y_{1}$ and $y_{2}$ by $y_{1}-y_{2}=$ : $z$ and substract them to get

$$
\nu|z|_{V}^{2}+b\left(y_{1}, y_{1}, z\right)-b\left(y_{2}, y_{2}, z\right)=\left(u_{1}-u_{2}, z\right)_{q, q^{\prime}}
$$

Since $b\left(y_{1}, z, z\right)=0$ because of $z, y_{1} \in V$, we can write

$$
b\left(y_{1}, y_{1}, z\right)-b\left(y_{2}, y_{2}, z\right)=b\left(y_{1}, y_{2}, z\right)-b\left(y_{1}, z, z\right)-b\left(y_{2}, y_{2}, z\right)=b\left(z, y_{2}, z\right)
$$


Then we obtain in view of $(3.5)$

$$
\left|b\left(y_{1}, y_{1}, z\right)-b\left(y_{2}, y_{2}, z\right)\right|=\left|b\left(z, y_{2}, z\right)\right| \leq C_{n}|z|_{4}^{2}\left|y_{2}\right|_{V} \leq C_{n} N_{4}^{2}|z|_{V}^{2} \frac{N_{q^{\prime}}}{\nu} \mathcal{M}_{q} \leq \frac{\nu}{2}|z|_{V}^{2}
$$

Finally, using Young's inequality again, we arrive at

$$
|z|_{V}^{2} \leq \frac{4 N_{q^{\prime}}^{2}}{\nu^{2}}\left|u_{1}-u_{2}\right|_{q}^{2}
$$

and the claim is proven.

To derive first-order necessary optimality conditions, we also need estimates of solutions of linearized equations.

Corollary 3.7. Let $\bar{y} \in V$ be the state corresponding to a control $\bar{u} \in U_{\text {ad }}$. Then for every $f \in V^{\prime}$ there exists a unique solution $y \in V$ of the linearized equation

$$
\nu(y, v)_{V}+b(y, \bar{y}, v)+b(\bar{y}, y, v)=\langle f, v\rangle \quad \forall v \in V .
$$

It holds

$$
|y|_{V} \leq \frac{2}{\nu}|f|_{V^{\prime}}
$$

Proof. Existence can be argued as in the proof of [28], Theorem II.1.2. Here it is necessary that $\bar{y}$ is the state associated to some control $\bar{u} \in U_{a d}$. In this case, we have some smallness property of $\bar{y}$ which ensures the solvability. The a priori estimate (3.8) can be shown along the lines of the previous proof.

\subsection{First order necessary optimality conditions}

So far, we provided results concerning the properties of the state equation. Now, we concentrate on the aspects of optimization. We denote by $G(u)=y$ the solution operator $u \mapsto y$ of the steady-state Navier-Stokes equations (3.3).

Lemma 3.8. The solution operator $G: L^{q}(\Omega)^{n} \mapsto V$ is Fréchet-differentiable. In particular, $G$ is Fréchetdifferentiable from $L^{2}(\Omega)^{n}$ to $V$. The derivative $G^{\prime}(u)$ is given by $G^{\prime}(\bar{u}) h=z$, where $z$ is a weak solution of

$$
\nu(z, v)_{V}+b(z, \bar{y}, v)+b(\bar{y}, z, v)=(h, v)_{2} \quad \forall v \in V
$$

with $\bar{u} \in U_{a d}, \bar{y}=G(\bar{u}), h \in L^{2}(\Omega)^{n}$.

Differentiability proofs of the solution mapping can be found in $[1,14,19]$.

Before discussing the second-order sufficient optimality condition, we derive for convenience the standard first-order necessary optimality condition.

Definition 3.9 (locally optimal control). A control $\bar{u} \in U_{a d}$ is called locally optimal in $L^{2}(\Omega)^{n}$, if there exists a constant $\rho>0$ such that

$$
J(\bar{y}, \bar{u}) \leq J\left(y_{h}, u_{h}\right)
$$

holds for all $u_{h} \in U_{a d}$ with $\left|\bar{u}-u_{h}\right|_{2} \leq \rho$. Here, $\bar{y}$ and $y_{h}$ denote the states associated to $\bar{u}$ and $u_{h}$, respectively.

In the presence of box constraints on the control it is straightforward to prove existence of optimal controls even with $\gamma=0$. For the unconstrained case one needs positivity of the parameter $\gamma$ to ensure this existence.

Theorem 3.10 (first-order necessary condition). Let $\bar{u}$ be a locally optimal control for (2.1) with associated state $\bar{y}=y(\bar{u})$. Then there exists a unique solution $\bar{\lambda} \in V$ of the adjoint equation

$$
\nu(\bar{\lambda}, v)_{V}+b(\bar{y}, v, \bar{\lambda})+b(v, \bar{y}, \bar{\lambda})=\left(\bar{y}-y_{d}, v\right)_{2} \quad \forall v \in V
$$


Moreover, the variational inequality

$$
(\gamma \bar{u}+\bar{\lambda}, u-\bar{u})_{2} \geq 0 \quad \forall u \in U_{a d}
$$

is satisfied.

Proof. We sketch the proof for convenience. Notice that we deal with the control-constrained case, i.e. $U_{a d} \neq$ $L^{2}(\Omega)^{2}$. The objective functional can be written as

$$
\phi(u)=J(\tilde{G}(u), u)=\frac{1}{2}\left|\tilde{G}(u)-y_{d}\right|_{2}^{2}+\frac{\gamma}{2}|u|_{2}^{2}
$$

where $\tilde{G}: L^{2}(\Omega)^{n} \mapsto V$ stands for the solution operator $G$ restricted to $L^{2}(\Omega)^{n}$. By Lemma 3.8, $\tilde{G}$ is also Fréchet-differentiable. The standard necessary condition for $\bar{u}$ to be a local optimum of $\phi(u)$ is $\phi^{\prime}(u)(u-\bar{u}) \geq 0$ for all $u \in U_{a d}$, i.e.

$$
\phi^{\prime}(u)(u-\bar{u})=\left(\tilde{G}(\bar{u})-y_{d}, \tilde{G}^{\prime}(\bar{u})(u-\bar{u})\right)_{2}+\gamma(\bar{u}, u-\bar{u})_{2} \geq 0 \quad \forall u \in U_{a d}
$$

We set $z:=\tilde{G}^{\prime}(\bar{u})(u-\bar{u})$, then $z$ satisfies the linear equation (3.9). Let $\bar{\lambda}$ be the solution of (3.10). Its existence can be reasoned like in Corollary 3.7. Testing (3.9) by $\bar{\lambda}$, we get

$$
\nu(z, \bar{\lambda})_{V}+b(z, \bar{y}, \bar{\lambda})+b(\bar{y}, z, \bar{\lambda})=(u-\bar{u}, \bar{\lambda})_{2} .
$$

Testing (3.10) by $z$ yields

$$
\nu(\bar{\lambda}, z)_{V}+b(\bar{y}, z, \bar{\lambda})+b(z, \bar{y}, \bar{\lambda})=\left(\bar{y}-y_{d}, z\right)_{2} .
$$

The left-hand sides in (3.13) and (3.14) are equal, so the right-hand sides are equal as well,

$$
(u-\bar{u}, \bar{\lambda})_{2}=\left(\bar{y}-y_{d}, z\right)_{2}=\left(\bar{y}-y_{d}, \tilde{G}^{\prime}(\bar{u})(u-\bar{u})\right)_{2} .
$$

Therefore, we obtain $\bar{\lambda}=\tilde{G}^{\prime}(\bar{u})^{*}\left(\bar{y}-y_{d}\right)=\tilde{G}^{\prime}(\bar{u})^{*}\left(\tilde{G}(\bar{u})-y_{d}\right)$. The variational inequality now reads,

$$
(\gamma \bar{u}+\bar{\lambda}, u-\bar{u})_{2} \geq 0 \quad \forall u \in U_{a d}
$$

hence, the claim is proven.

The solution $\bar{\lambda}$ of the adjoint equation (3.10) is said to be the adjoint state associated with $\bar{y}$. It can be easily verified that $\bar{\lambda}$ is a weak solution of the adjoint partial differential equation

$$
\begin{aligned}
-\nu \Delta \lambda-(\bar{y} \cdot \nabla) \lambda+(\nabla \bar{y})^{T} \lambda+\nabla \mu & =\bar{y}-y_{d} & & \text { on } \Omega, \\
\operatorname{div} \lambda & =0 & & \text { on } \Omega, \\
\lambda & =0 & & \text { on } \Gamma .
\end{aligned}
$$

The function $\mu$ might be interpreted as the adjoint pressure.

Corollary 3.11. The adjoint state $\bar{\lambda}$, given by (3.10), satisfies

$$
|\bar{\lambda}|_{V} \leq \frac{2}{\nu} N_{2}\left|\bar{y}-y_{d}\right|_{2}
$$

Proof. The estimate can be proven along the lines of Lemma 3.6. 
To simplify notations we denote the pair $(y, u)$ by $v$. It is called admissible, if $u$ belongs to $U_{a d}$ and $y$ is the weak solution of (2.2) associated with $u$.

Let us introduce the Lagrange function $\mathcal{L}: V \times L^{2}(\Omega)^{n} \times V \mapsto \mathbb{R}$ for the optimal control problem as follows:

$$
\mathcal{L}(y, u, \lambda)=J(u, y)-\nu(y, \lambda)_{V}-b(y, y, \lambda)+(u, \lambda)_{2} .
$$

This function is twice Fréchet-differentiable with respect to $u$ and $y$. The reader can readily verify that the necessary conditions can be expressed equivalently by

$$
\mathcal{L}_{y}(\bar{y}, \bar{u}, \bar{\lambda}) y=0 \quad \forall y \in V
$$

and

$$
\mathcal{L}_{u}(\bar{y}, \bar{u}, \bar{\lambda})(u-\bar{u}) \geq 0 \quad \forall u \in U_{a d}
$$

Here, $\mathcal{L}_{y}, \mathcal{L}_{u}$ denote the partial Fréchet-derivative of $\mathcal{L}$ with respect to $y$ and $u$. The Fréchet-differentiability of $\mathcal{L}$ is shown in the next Lemma.

Lemma 3.12. The Lagrangian $\mathcal{L}$ is twice Fréchet-differentiable with respect to $v=(y, u)$ from $V \times L^{2}(\Omega)^{n}$ to $\mathbb{R}$. The second-order derivative at $\bar{v}=(\bar{y}, \bar{u})$ fulfills together with the associated adjoint state $\bar{\lambda}$

$$
\mathcal{L}_{v v}(\bar{v}, \bar{\lambda})\left[\left(z_{1}, h_{1}\right),\left(z_{2}, h_{2}\right)\right]=\mathcal{L}_{u u}(\bar{v}, \bar{\lambda})\left[h_{1}, h_{2}\right]+\mathcal{L}_{y y}(\bar{v}, \bar{\lambda})\left[z_{1}, z_{2}\right]
$$

and

$$
\left|\mathcal{L}_{y y}(\bar{v}, \bar{\lambda})\left[z_{1}, z_{2}\right]\right| \leq c_{\mathcal{L}}\left|z_{1}\right|_{V}\left|z_{2}\right|_{V}
$$

for all $\left(z_{i}, h_{i}\right) \in V \times L^{2}(\Omega)^{n}$ with some constant $c_{\mathcal{L}}>0$ that does not depend on $\bar{v}, z_{1}, z_{2}$.

Proof. The first-order derivatives of $\mathcal{L}$ with respect to $y$ and $u$ are

$$
\begin{aligned}
& \mathcal{L}_{y}(\bar{v}, \bar{\lambda}) z=\left(z, \bar{y}-y_{d}\right)_{2}-\nu(z, \bar{\lambda})_{V}-b(z, \bar{y}, \bar{\lambda})-b(\bar{y}, z, \bar{\lambda}) \\
& \mathcal{L}_{u}(\bar{v}, \bar{\lambda}) h=\gamma(h, \bar{u})_{2}+(u, \bar{\lambda})_{2} .
\end{aligned}
$$

The mappings $\bar{y} \mapsto \mathcal{L}_{y}(\bar{v}, \bar{\lambda})$ and $\bar{u} \mapsto \mathcal{L}_{u}(\bar{v}, \bar{\lambda})$ are affine linear. Their linear parts are bounded, hence continuous. Therefore, both mappings are Fréchet-differentiable. This shows that $\mathcal{L}$ is twice Fréchet-differentiable as well. The second-order derivative of $\mathcal{L}$ with respect to $v$ is

$$
\begin{aligned}
\mathcal{L}_{v v}(\bar{v}, \bar{\lambda})\left[\left(z_{1}, h_{1}\right)\left(z_{2}, h_{2}\right)\right] & =\mathcal{L}_{u u}(\bar{v}, \bar{\lambda})\left[h_{1}, h_{2}\right]+\mathcal{L}_{y y}(\bar{v}, \bar{\lambda})\left[z_{1}, z_{2}\right] \\
& =\gamma\left(h_{1}, h_{2}\right)_{2}+\left(z_{1}, z_{2}\right)_{2}-b\left(z_{1}, z_{2}, \bar{\lambda}\right)-b\left(z_{2}, z_{1}, \bar{\lambda}\right),
\end{aligned}
$$

since mixed derivatives do not appear. Then we can estimate

$$
\begin{aligned}
\left|\mathcal{L}_{y y}(\bar{v}, \bar{\lambda})\left[z_{1}, z_{2}\right]\right| & \leq\left|z_{1}\right|_{2}\left|z_{2}\right|_{2}+\left|b\left(z_{1}, z_{2}, \bar{\lambda}\right)\right|+\left|b\left(z_{2}, z_{1}, \bar{\lambda}\right)\right| \\
& \leq\left|z_{1}\right|_{2}\left|z_{2}\right|_{2}+2 C_{n}\left|z_{1}\right|_{4}\left|z_{2}\right|_{4}|\bar{\lambda}|_{V} \\
& \leq\left.\left. c_{\mathcal{L}}\left|z_{1}\right|_{V}\right|_{2}\right|_{V} .
\end{aligned}
$$

Here we used the estimates of $b$ in Lemma 3.3 and the boundedness of the adjoint state, see Corollary 3.11.

The Lagrangian has only non-zero derivatives up to order two. Derivatives of higher order vanish. Therefore, it holds

$$
\mathcal{L}(y+z, u+h)=\mathcal{L}(y, u)+\mathcal{L}_{y}(y, u) z+\mathcal{L}_{u}(y, u) h+\frac{1}{2} \mathcal{L}_{y y}(y, u)[z, z]+\frac{1}{2} \mathcal{L}_{u u}(y, u)[h, h] .
$$

A remainder term does not appear. To shorten notations, we abbreviate $[v, v]$ by $[v]^{2}$, i.e.

$$
\mathcal{L}_{v v}(\bar{v}, \bar{\lambda})[(z, h)]^{2}:=\mathcal{L}_{v v}(\bar{v}, \bar{\lambda})[(z, h),(z, h)]
$$




\subsection{Second-order sufficient optimality condition}

In the following, $\bar{v}=(\bar{y}, \bar{u})$ is a fixed admissible reference pair. We suppose that the first-order necessary optimality conditions are fulfilled at $\bar{v}$.

Definition 3.13 (strongly active sets). For fixed $\varepsilon>0$ and all $i=1, \ldots, n$ we define sets $\Omega_{\varepsilon, i}$ by

$$
\Omega_{\varepsilon, i}=\left\{x \in \Omega:\left|\gamma \bar{u}_{i}(x)+\bar{\lambda}_{i}(x)\right|>\varepsilon\right\}
$$

Here, $v_{i}(x)$ denotes the value of the $i$-th component of a vector function $v \in V$ at $x \in \Omega$. Since $\bar{u}$ and $\bar{\lambda}$ are measurable functions, the sets $\Omega_{\varepsilon, i}$ are measurable, too. Moreover, for $u \in L^{p}(\Omega)^{n}$ and $1 \leq p<\infty$ we define the $L^{p}$-norm with respect to the set of positivity by

$$
|u|_{L^{p}, \Omega_{\varepsilon}}:=\left(\sum_{i=1}^{n}\left|u_{i}\right|_{L^{p}\left(\Omega_{\varepsilon, i}\right)}^{p}\right)^{1 / p}
$$

Notice that the variational inequality (3.11) uniquely determines $\bar{u}_{i}$ on $\Omega_{\varepsilon, i}$. If $\gamma \bar{u}_{i}(x)+\bar{\lambda}_{i}(x) \geq \varepsilon$ then $\bar{u}_{i}(x)=$ $u_{a}(x)$ must hold. On the other hand, it follows $\bar{u}_{i}(x)=u_{b}(x)$, if $\gamma \bar{u}_{i}(x)+\bar{\lambda}_{i}(x) \leq-\varepsilon$ is satisfied.

Corollary 3.14. It holds

$$
\sum_{i=1}^{n} \int_{\Omega_{\varepsilon, i}}\left(\gamma \bar{u}_{i}(x)+\bar{\lambda}_{i}(x)\right)\left(u_{i}(x)-\bar{u}_{i}(x)\right) \mathrm{d} x \geq \varepsilon|u-\bar{u}|_{L^{1}, \Omega_{\varepsilon}}
$$

for all $u \in U_{a d}$.

Proof. From the variational inequality (3.11) we conclude the pointwise condition

$$
\left(\gamma \bar{u}_{i}(x)+\bar{\lambda}_{i}(x)\right)\left(u_{i}(x)-\bar{u}_{i}(x)\right) \geq 0
$$

for almost all $x \in \Omega, i=1, \ldots, n$. Therefore,

$$
\begin{aligned}
\int_{\Omega_{\varepsilon, i}}\left(\gamma \bar{u}_{i}(x)+\bar{\lambda}_{i}(x)\right)\left(u_{i}(x)-\bar{u}_{i}(x)\right) \mathrm{d} x & =\int_{\Omega_{\varepsilon, i}}\left|\gamma \bar{u}_{i}(x)+\bar{\lambda}_{i}(x)\right| \mid\left(u_{i}(x)-\bar{u}_{i}(x) \mid \mathrm{d} x\right. \\
& \geq \varepsilon \int_{\Omega_{\varepsilon, i}} \mid\left(u_{i}(x)-\bar{u}_{i}(x) \mid \mathrm{d} x\right. \\
& =\varepsilon|u-\bar{u}|_{L^{1}\left(\Omega_{\varepsilon, i}\right)}
\end{aligned}
$$

is satisfied. The claim follows by summing up this expression over $i=1, \ldots, n$. 
We shall assume that the optimal pair $\bar{v}=(\bar{y}, \bar{u})$ and the associated adjoint state $\bar{\lambda}$ satisfy the following coercivity assumption on $\mathcal{L}^{\prime \prime}(\bar{v}, \bar{\lambda})$, henceforth called second-order sufficient optimality condition:

$$
\begin{aligned}
& (\mathbf{S S C})\left\{\begin{array}{l}
\text { There exist } \varepsilon>0 \text { and } \delta>0 \text { such that } \\
\qquad \mathcal{L}_{v v}(\bar{v}, \bar{\lambda})[(z, h)]^{2} \geq \delta|h|_{q}^{2} \\
\text { holds for all pairs }(z, h) \in V \times L^{2}(\Omega)^{n} \text { with } \\
h=u-\bar{u}, u \in U_{a d}, h_{i}=0 \text { on } \Omega_{\varepsilon, i} \text { for } i=1, \ldots, n, \\
\text { and } z \in V \text { being the weak solution of the linearized equation } \\
\qquad \nu(z, w)_{V}+b(\bar{y}, z, w)+b(z, \bar{y}, w)=(h, w)_{2} \quad \forall w \in V . \\
\text { Remark parameter } q \text { is chosen according to Assumption }(A 1) .
\end{array}\right. \\
& \bar{u}(x)=u_{b}(x) \text {. The condition } \varepsilon>0 \text { can not be relaxed to } \varepsilon=0, \text { see the } \operatorname{counterexample~in~}[16] .
\end{aligned}
$$

Remark 3.16. Second-order sufficient conditions cannot be expected if no penalty term is present, which corresponds to the case $\gamma=0$. For instance, it was shown in [29, Lem. 5.1] that (SSC) necessarily implies $\gamma>0$.

Next we will prove that (SSC), together with the first-order necessary conditions, is sufficient for local optimality of $(\bar{y}, \bar{u})$.

Theorem 3.17. Let $\bar{v}=(\bar{y}, \bar{u})$ be admissible for the optimal control problem and suppose that $\bar{v}$ fulfills the first-order necessary optimality condition with associated adjoint state $\bar{\lambda}$. Assume further that (SSC) is satisfied at $\bar{v}$. Then there exist $\alpha>0$ and $\rho>0$ such that

$$
J(v) \geq J(\bar{v})+\alpha|u-\bar{u}|_{q}^{2}
$$

holds for all admissible pairs $v=(y, u)$ with $|u-\bar{u}|_{s} \leq \rho$. The exponents $s$ and $q$ are chosen such that the Assumptions (A1) and (A2) are met.

Proof. Throughout the proof, $c$ is used as a generic constant. Suppose that $\bar{v}$ fulfills the assumptions of the theorem. Let $(y, u)$ be another admissible pair. We have

$$
J(\bar{v})=\mathcal{L}(\bar{v}, \bar{\lambda}) \text { and } J(v)=\mathcal{L}(v, \bar{\lambda}),
$$

since $\bar{v}$ and $v$ are admissible. Taylor-expansion of the Lagrange-function yields

$$
\mathcal{L}(v, \bar{\lambda})=\mathcal{L}(\bar{v}, \bar{\lambda})+\mathcal{L}_{y}(\bar{v}, \bar{\lambda})(y-\bar{y})+\mathcal{L}_{u}(\bar{v}, \bar{\lambda})(u-\bar{u})+\frac{1}{2} \mathcal{L}_{v v}(\bar{v}, \bar{\lambda})[v-\bar{v}, v-\bar{v}]
$$

Notice that there is no remainder term due to the quadratic nature of the nonlinearities. Moreover, the necessary conditions (3.15), (3.16) are satisfied at $\bar{v}$ with adjoint state $\bar{\lambda}$. Therefore, the second term vanishes. The third term is nonnegative due to the variational inequality (3.16). However, we get even more by Corollary 3.14,

$$
\begin{aligned}
\mathcal{L}_{u}(\bar{v}, \bar{\lambda})(u-\bar{u}) & =\int_{\Omega}(\gamma \bar{u}+\bar{\lambda})(u-\bar{u}) \mathrm{d} x \\
& \geq \sum_{i=1}^{n} \int_{\Omega_{\varepsilon, i}}\left(\gamma \bar{u}_{i}+\bar{\lambda}_{i}\right)\left(u_{i}-\bar{u}_{i}\right) \mathrm{d} x \geq \varepsilon|u-\bar{u}|_{L^{1}, \Omega_{\varepsilon}},
\end{aligned}
$$


confer the Definition 3.13 of $|\cdot|_{L^{1}, \Omega_{\varepsilon}}$. So we arrive at

$$
\begin{aligned}
J(v) & =J(\bar{v})+\mathcal{L}_{y}(\bar{v}, \bar{\lambda})(y-\bar{y})+\mathcal{L}_{u}(\bar{v}, \bar{\lambda})(u-\bar{u})+\frac{1}{2} \mathcal{L}_{v v}(\bar{v}, \bar{\lambda})[v-\bar{v}]^{2} \\
& \geq J(\bar{v})+\varepsilon|u-\bar{u}|_{L^{1}, \Omega_{\varepsilon}}+\frac{1}{2} \mathcal{L}_{v v}(\bar{v}, \bar{\lambda})[v-\bar{v}]^{2} .
\end{aligned}
$$

Next, we investigate the second derivative of $\mathcal{L}$. Here, we invoke assumption $(3.18)$ on the coercitivity of $\mathcal{L}_{v v}$ on a certain subspace. To do so, we introduce a new admissible control $\tilde{u} \in L^{s}(\Omega)^{n}$ by

$$
\tilde{u}_{i}(x)=\left\{\begin{array}{ll}
\bar{u}_{i}(x) & \text { on } \Omega_{\varepsilon, i} \\
u_{i}(x) & \text { on } \Omega \backslash \Omega_{\varepsilon, i}
\end{array} \text { for } i=1, \ldots, n .\right.
$$

Then, we have $u-\bar{u}=(u-\tilde{u})+(\tilde{u}-\bar{u})$, where $(u-\tilde{u})_{i}=0$ on $\Omega \backslash \Omega_{\varepsilon, i}$ and $(\tilde{u}-\bar{u})_{i}=0$ on $\Omega_{\varepsilon, i}$, so that $h:=\tilde{u}-\bar{u}$ fits in the assumptions of (SSC). The difference $z:=y-\bar{y}$ solves the equation

$$
\nu(z, w)_{V}+b(z, \bar{y}, w)+b(\bar{y}, z, w)=(u-\bar{u}, w)_{q, q^{\prime}}-b(y-\bar{y}, y-\bar{y}, w) \quad \forall w \in V .
$$

We split $z=y-\bar{y}$ into $y_{h}+y_{r}$, where $y_{r}$ and $y_{h}$ solve the equations

$$
\nu\left(y_{h}, w\right)_{V}+b\left(y_{h}, \bar{y}, w\right)+b\left(\bar{y}, y_{h}, w\right)=(h, w)_{q, q^{\prime}} \quad \forall w \in V
$$

and

$$
\nu\left(y_{r}, w\right)_{V}+b\left(y_{r}, \bar{y}, w\right)+b\left(\bar{y}, y_{r}, w\right)=(u-\tilde{u}, w)_{q, q^{\prime}}-b(y-\bar{y}, y-\bar{y}, w) \quad \forall w \in V .
$$

Notice that (3.22) is linear and that $\left(y_{h}, h\right)$ belongs to the subspace where (SSC) applies. The norm of these auxiliary states has to be estimated. Using Lemma 3.6 we obtain

$$
\left|y_{h}\right|_{V} \leq c|h|_{q} \leq c\left(|\tilde{u}-u|_{q}+|u-\bar{u}|_{q}\right) .
$$

To estimate $\left|y_{r}\right|_{V}$, we have to investigate the $V^{\prime}$-norm of the right-hand side in (3.23), which defines a linear continuous functional on $V$. By Lemma 3.3 we find

$$
|b(y-\bar{y}, y-\bar{y}, \cdot)|_{V^{\prime}} \leq C_{n} N_{4}^{2}|y-\bar{y}|_{V}^{2} .
$$

Now we apply Corollary 3.7 and get

$$
\left|y_{r}\right|_{V} \leq \frac{2}{\nu}\left(C_{n} N_{4}^{2}|y-\bar{y}|_{V}^{2}+N_{q^{\prime}}|u-\tilde{u}|_{q}\right) \leq c\left(|u-\bar{u}|_{q}^{2}+|u-\tilde{u}|_{q}\right) .
$$

Denote the pair $\left(y_{h}, h\right)$ by $v_{h}$. This pair fits in the assumptions of the theorem. We continue the investigation of the Lagrangian by

$$
\begin{aligned}
\mathcal{L}_{v v}(\bar{v}, \bar{\lambda})[v-\bar{v}]^{2}= & \mathcal{L}_{u u}(\bar{v}, \bar{\lambda})[u-\tilde{u}+h]^{2}+\mathcal{L}_{y y}(\bar{v}, \bar{\lambda})\left[y_{h}+y_{r}\right]^{2} \\
= & \mathcal{L}_{v v}(\bar{v}, \bar{\lambda})\left[v_{h}\right]^{2}+2 \mathcal{L}_{u u}(\bar{v}, \bar{\lambda})[u-\tilde{u}, h] \\
& +\mathcal{L}_{u u}(\bar{v}, \bar{\lambda})[u-\tilde{u}]^{2}+2 \mathcal{L}_{y y}(\bar{v}, \bar{\lambda})\left[y_{r}, y_{h}\right]+\mathcal{L}_{y y}(\bar{v}, \bar{\lambda})\left[y_{r}\right]^{2} .
\end{aligned}
$$

(SSC) applies to the first term $\mathcal{L}_{v v}(\bar{v}, \bar{\lambda})\left[v_{h}\right]^{2}$. The second-order derivative with respect to $u$ satisfies

$$
2 \mathcal{L}_{u u}(\bar{v}, \bar{\lambda})[u-\tilde{u}, h]+\mathcal{L}_{u u}(\bar{v}, \bar{\lambda})[u-\tilde{u}]^{2}=2 \gamma(u-\tilde{u}, h)_{2}+\gamma|u-\tilde{u}|_{2}^{2} .
$$


By definition of $\tilde{u}$ we know that $(u-\tilde{u})_{i}$ vanishes on $\Omega \backslash \Omega_{\varepsilon, i}$ whereas $(h)_{i}$ vanishes on $\Omega_{\varepsilon, i}$. So their scalar product is zero. Therefore, it holds

$$
2 \mathcal{L}_{u u}(\bar{v}, \bar{\lambda})[u-\tilde{u}, h]+\mathcal{L}_{u u}(\bar{v}, \bar{\lambda})[u-\tilde{u}]^{2}=\gamma|u-\bar{u}|_{L^{2}, \Omega_{\varepsilon}}^{2} \geq 0 .
$$

The remaining terms in (3.26) are treated by Lemma 3.12 and the estimates (3.24), (3.25),

$$
\begin{aligned}
\left|2 \mathcal{L}_{y y}(\bar{v}, \bar{\lambda})\left[y_{r}, y_{h}\right]+\mathcal{L}_{y y}(\bar{v}, \bar{\lambda})\left[y_{r}, y_{r}\right]\right| & \leq c\left(\left|y_{h}\right|_{V}\left|y_{r}\right|_{V}+\left|y_{r}\right|_{V}^{2}\right) \\
& \leq c\left\{\left(|\tilde{u}-u|_{q}+|u-\bar{u}|_{q}\right)\left(|u-\bar{u}|_{q}^{2}+|u-\tilde{u}|_{q}\right)+|u-\bar{u}|_{q}^{4}+|u-\tilde{u}|_{q}^{2}\right\}
\end{aligned}
$$

Now we can proceed with the investigation of $\mathcal{L}_{v v}$ in (3.26). Invoking (3.27) and (3.28), we obtain from (3.26)

$$
\mathcal{L}_{v v}(\bar{v}, \bar{\lambda})[v-\bar{v}]^{2} \geq \delta|h|_{q}^{2}-c\left\{\left(|\tilde{u}-u|_{q}+|u-\bar{u}|_{q}\right)\left(|u-\bar{u}|_{q}^{2}+|u-\tilde{u}|_{q}\right)+|u-\bar{u}|_{q}^{4}+|u-\tilde{u}|_{q}^{2}\right\} .
$$

Our next aim is to eliminate $h$ such that only terms containing $u-\bar{u}$ and $\tilde{u}-u$ appear. To achieve this goal, we first notice that

which immediately gives

$$
|u-\bar{u}|_{q}^{2}=|u-\tilde{u}+h|_{q}^{2} \leq 2\left(|u-\tilde{u}|_{q}^{2}+|h|_{q}^{2}\right)
$$

$$
|h|_{q}^{2} \geq \frac{1}{2}|u-\bar{u}|_{q}^{2}-|u-\tilde{u}|_{q}^{2}
$$

Applying Young's inequality several times to separate the powers of $|u-\bar{u}|_{q}$ and $|\tilde{u}-u|_{q}$ we get from (3.29)

$$
\begin{aligned}
\mathcal{L}_{v v}(\bar{v}, \bar{\lambda})[v-\bar{v}]^{2} \geq & \frac{\delta}{2}|u-\bar{u}|_{q}^{2}-c\left\{|u-\bar{u}|_{q}^{4}+|u-\bar{u}|_{q}^{3}+|u-\bar{u}|_{q}^{2}|\tilde{u}-u|_{q}\right. \\
& \left.+|u-\bar{u}|_{q}|\tilde{u}-u|_{q}+|\tilde{u}-u|_{q}^{2}\right\} \\
\geq & \frac{\delta}{4}|u-\bar{u}|_{q}^{2}-c\left\{|u-\bar{u}|_{q}^{4}+|u-\bar{u}|_{q}^{3}+|\tilde{u}-u|_{q}^{2}\right\} \\
\geq & |u-\bar{u}|_{q}^{2}\left(\frac{\delta}{4}-c\left\{|u-\bar{u}|_{q}^{2}+|u-\bar{u}|_{q}\right\}\right)-c|\tilde{u}-u|_{q}^{2} .
\end{aligned}
$$

If $u$ is sufficiently close to $\bar{u}$, i.e. $|u-\bar{u}|_{q} \leq N_{s, q}|u-\bar{u}|_{s} \leq N_{s, q} \rho_{1}$, then the term in brackets is greater than $\delta / 8$. Hence we arrive at

$$
\mathcal{L}_{v v}(\bar{v}, \bar{\lambda})[v-\bar{v}]^{2} \geq \frac{\delta}{8}|u-\bar{u}|_{q}^{2}-c|\tilde{u}-u|_{q}^{2}
$$

Now we are able to complete the estimation of the objective functional. We continue (3.20) by

$$
\begin{aligned}
J(v) & \geq J(\bar{v})+\varepsilon|u-\bar{u}|_{L^{1}, \Omega_{\varepsilon}}+\frac{1}{2} \mathcal{L}_{v v}(\bar{v}, \bar{\lambda})[v-\bar{v}]^{2} \\
& \geq J(\bar{v})+\varepsilon|u-\bar{u}|_{L^{1}, \Omega_{\varepsilon}}+\frac{\delta}{16}|u-\bar{u}|_{q}^{2}-\frac{c}{2}|\tilde{u}-u|_{q}^{2} .
\end{aligned}
$$

By definition, $\tilde{u}$ and $u$ differ only on the sets $\Omega \backslash \Omega_{i, \varepsilon}$, while $\tilde{u}$ and $\bar{u}$ coincide on $\Omega \backslash \Omega_{i, \varepsilon}$, hence we conclude using Lemma 3.1,

$$
|\tilde{u}-u|_{q}^{2} \leq|\tilde{u}-u|_{1}|\tilde{u}-u|_{s}=|u-\bar{u}|_{L^{1}, \Omega_{\varepsilon}}|u-\bar{u}|_{s} \leq \rho_{2}|u-\bar{u}|_{L^{1}, \Omega_{\varepsilon}},
$$

if the $L^{s}$-norm of the difference is sufficiently small, i.e. $|u-\bar{u}|_{s} \leq \rho_{2}$. Hence,

$$
\begin{aligned}
J(v) & \geq J(\bar{v})+\varepsilon|u-\bar{u}|_{L^{1}, \Omega_{\varepsilon}}+\frac{\delta}{16}|u-\bar{u}|_{q}^{2}-\frac{c}{2}|\tilde{u}-u|_{q}^{2} \\
& \geq J(\bar{v})+\left(\varepsilon-\frac{c}{2} \rho_{2}\right)|u-\bar{u}|_{L^{1}, \Omega_{\varepsilon}}+\frac{\delta}{16}|u-\bar{u}|_{q}^{2} .
\end{aligned}
$$


Choosing $\rho_{2}$ so small that $\varepsilon-\frac{c}{2} \rho_{2}>0$, we prove the claim with $\alpha=\delta / 16$ and $\rho=\min \left(\rho_{1}, \rho_{2}\right)$.

The next result is a immediate conclusion.

Theorem 3.18. Suppose that the assumptions of Theorem 3.17 hold true. Then $\bar{u}$ is a locally optimal control in the sense of $L^{s}(\Omega)^{n}$.

Remark 3.19. The second-order sufficient optimality condition can be adapted to general objective functionals following [10]. However, then one obtains differentiability of the functional $J$ with respect to control and state only in $L^{\infty}$-type spaces. Consequently, one has to work with $L^{\infty}$-neighborhoods of the reference control.

\subsection{Extensions and remarks}

Let us investigate briefly under which conditions it can be ensured that condition (SSC) holds. For simplicity, we consider the unconstrained case, $U_{a d}=L^{2}(\Omega)^{2}$. It is well-known that the second-order sufficient condition is satisfied if the residual $\bar{y}-y_{d}$ is small enough, i.e. the desired state could be approximated closely. See for instance $[21,22]$. Thanks to the precise estimates of the solutions of the nonlinear as well as the linear systems in the previous sections, we can specify how small the difference between optimal state and desired state has to be.

Lemma 3.20. Let $\bar{v}=(\bar{y}, \bar{u})$ be admissible for the optimal control problem and suppose that $\bar{v}$ fulfills the first-order necessary optimality condition with associated adjoint state $\bar{\lambda}$. Then the second-order sufficient condition (SSC) is fulfilled if the residual $\left|\bar{y}-y_{d}\right|_{2}$ is sufficiently small or the parameters $\gamma$ and $\nu$ are sufficiently large, such that the inequality (3.30) below is satisfied.

Proof. The second derivative of the Lagrangian $\mathcal{L}$ is given by

$$
\mathcal{L}_{v v}(\bar{v}, \bar{\lambda})[(z, h)]^{2}=|z|_{2}^{2}+\gamma|h|_{2}^{2}+2 b(z, z, \bar{\lambda})
$$

The only addend that can disturb positivity is $b(z, z, \bar{\lambda})$. We can estimate it according to (3.2) by

$$
|b(z, z, \bar{\lambda})| \leq C_{n} N_{4}^{2}|z|_{V}^{2}|\bar{\lambda}|_{V}
$$

The state $z$ and the adjoint $\bar{\lambda}$ are weak solutions of linear equations. Hence they can be estimated using Corollaries 3.7 and 3.11 by

$$
|z|_{V} \leq \frac{2}{\nu} N_{q^{\prime}}|h|_{q}, \quad|\bar{\lambda}|_{V} \leq \frac{2}{\nu} N_{2}\left|\bar{y}-y_{d}\right|_{2}
$$

Thus, we obtain

$$
\begin{aligned}
\mathcal{L}_{v v}(\bar{v}, \bar{\lambda})[(z, h)]^{2} & \geq|z|_{2}^{2}+\gamma|h|_{2}^{2}-2\left(\frac{2}{\nu} N_{q^{\prime}}|h|_{q}\right)^{2}\left(\frac{2}{\nu} N_{2}\left|\bar{y}-y_{d}\right|_{2}\right) \\
& \geq|z|_{2}^{2}+\left(\gamma N_{2, q}-\frac{16}{\nu^{3}} N_{q^{\prime}}^{2} N_{2}\left|\bar{y}-y_{d}\right|_{2}\right)|h|_{q}^{2}
\end{aligned}
$$

with $N_{2, q}=\operatorname{vol}(\Omega)^{1 / q-1 / 2}$. This implies that $\mathcal{L}_{v v}$ is positive definite, if it holds

$$
\gamma>\frac{16}{\nu^{3}} \frac{N_{q^{\prime}}^{2} N_{2}}{N_{2, q}}\left|\bar{y}-y_{d}\right|_{2},
$$

which means that $\gamma, \nu$ are large enough or $\left|\bar{y}-y_{d}\right|_{2}$ is small enough. Please note, that the term $|z|_{2}^{2}$ cannot provide any additional coercivity since the mapping $h \mapsto z$ is compact from $L^{q}(\Omega)^{2}$ to $L^{2}(\Omega)^{2}$. The constants $N$ are imbedding constants depending only on the domain $\Omega$. 
Here, we presented a condition on the parameters under which (SSC) is satisfied. However, it cannot be answered by analytic considerations in what flow configurations, e.g. cavity or channel flow, it is likely that (SSC) is fulfilled.

\subsubsection{General objective functionals}

The analysis of the proof of sufficiency in not restricted to the special quadratic nature of the objective functional $J$ defined in (2.1). Let us consider the minimization of the functional

$$
\tilde{J}(y, u)=\int_{\Omega} q(x, y(x), u(x)) \mathrm{d} x
$$

We have to require appropriate measurability and differentiability assumptions, which are standard in the literature, see for instance [10,25]. Furthermore, we need $L^{\infty}$-regularity of the state and control to obtain Frechét differentiability of the objective functional. Additionally, we get an extra second-order remainder term in the Taylor expansion (3.20) of the Lagrange functional. Up to this differences, the method of proof remains the same.

\subsubsection{An equivalent formulation of second-order sufficient optimality conditions}

Here, we comment on other formulations of second-order suficient conditions known from literature [4,5,8]. Let us consider the sufficient optimality condition (SSC) with parameters $q=q^{\prime}=2$. We assume in this section that the Assumptions (A1) and (A2) are satisfied. Let us recall (SSC) for $q=2$ for convenience:

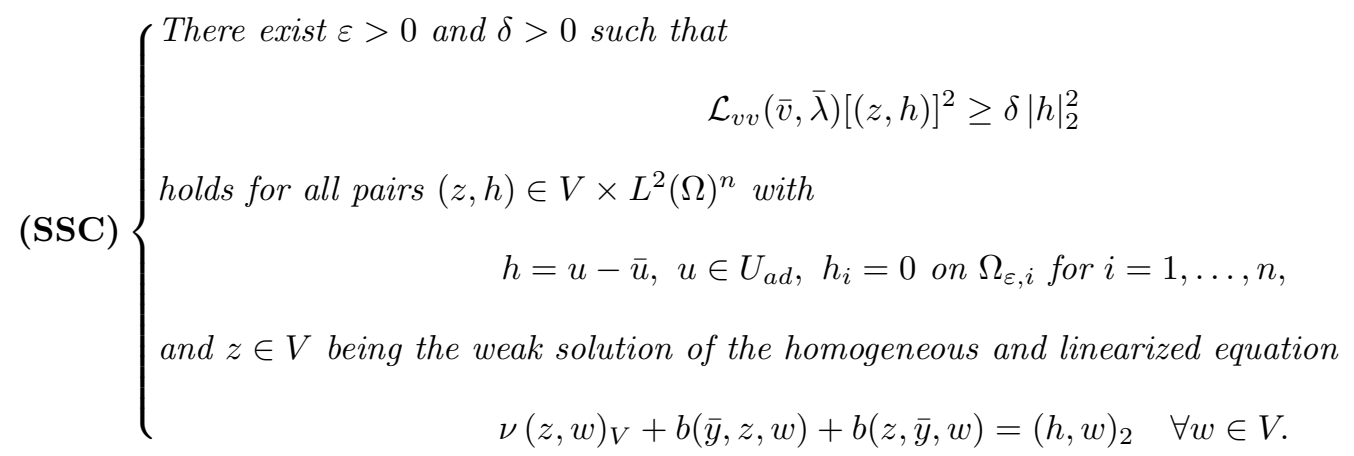

In the case of quadratic objective functionals, the condition (SSC) can be simplified equivalently following the lines of Bonnans $[4,5]$. The equivalent condition introduced there was discussed for semilinear elliptic equations but not yet for control of Navier-Stokes equations.

The tangent cone on $U_{a d}$ at $\bar{u}$, denoted by $T(\bar{u})$, is defined by

$$
T(\bar{u})=\left\{h \in L^{2}(\Omega)^{n} \mid h=\lim _{k \rightarrow \infty} \frac{u_{k}-\bar{u}}{t_{k}}, u_{k} \in U_{a d}, t_{k} \downarrow 0\right\} .
$$


$T(\bar{u})$ is convex, non-empty and closed in $L^{2}(\Omega)^{n}$, hence also weakly closed. By $T(\bar{u})$, we are able to formulate (SSC) in the following way:

$\left(\mathbf{S S C}_{0}\right)\left\{\begin{array}{c}\text { It holds } \\ \left.\text { for all pairs }(z, h) \in V \times L^{2}(\Omega)^{n} \text { with } h \not \equiv, \bar{v}, \bar{\lambda}\right)[(z, h)]^{2}>0 \\ h_{i}=0 \text { on } \Omega_{0, i} \\ \text { for all } i=1, \ldots, n, \text { where } z \text { is the solution of the associated linearized equation (3.33). }\end{array}\right.$

Notice that $\Omega_{0, i}=\left\{x \in \Omega:\left|\gamma \bar{u}_{i}(x)+\bar{\lambda}_{i}(x)\right|>0\right\}$.

Theorem 3.21. The conditions $(S S C)$ and $\left(S S C_{0}\right)$ are equivalent.

Proof. It is easy to see that (SSC) implies ( $\left.\mathrm{SSC}_{0}\right)$. Let $0 \neq h \in T(\bar{u})$ with $h_{i}=0$ a.e. on $\Omega_{0, i}$. Since $\Omega_{\varepsilon, i} \subset \Omega_{0, i}$, it holds $h_{i}=0$ on $\Omega_{\varepsilon, i}$. Further, there exists a sequence $h_{k}=\left(u_{k}-\bar{u}\right) / t_{k}$ converging to $h$ in $L^{2}(\Omega)^{n}$. After extracting a subsequence if necessary, we find that $u_{k, i}(x)-\bar{u}_{i}(x) \rightarrow 0$ a.e. on $\Omega_{0, i}$. Hence, we can choose $u_{k}$ such that $u_{k, i}(x)=\bar{u}_{i}(x)$ on $\Omega_{0, i}$. This implies $h_{k, i}=0$ on $\Omega_{0, i}$, and $h_{k}$ can be used as test function in (SSC). Let $z, z_{k}$ be the associated solutions of the linearized equation and $v:=(z, h), v_{k}:=\left(z_{k}, h_{k}\right)$. Then it holds

$$
\begin{aligned}
\mathcal{L}_{v v}(\bar{v}, \bar{\lambda})[v]^{2} & =\mathcal{L}_{v v}(\bar{v}, \bar{\lambda})\left[v-v_{k}+v_{k}\right]^{2} \\
& =\mathcal{L}_{v v}(\bar{v}, \bar{\lambda})\left[v_{k}\right]^{2}+2 \mathcal{L}_{v v}(\bar{v}, \bar{\lambda})\left[v-v_{k}, v_{k}\right]+L_{v v}(\bar{v}, \bar{\lambda})\left[v-v_{k}\right]^{2} .
\end{aligned}
$$

Using assumption (3.31), estimates of $\mathcal{L}_{v v}$ in Lemma 3.12, and Corollary 3.7, we obtain

$$
\mathcal{L}_{v v}(\bar{v}, \bar{\lambda})[v]^{2} \geq \delta\left|h_{k}\right|_{2}^{2}-c_{1}\left|h-h_{k}\right|_{2}\left|h_{k}\right|_{2}-c_{2}\left|h-h_{k}\right|_{2}^{2} \geq \frac{\delta}{2}\left|h_{k}\right|_{2}^{2}-c\left|h-h_{k}\right|_{2}^{2},
$$

which gives in the limit $k \rightarrow \infty$

and $\left(\mathrm{SSC}_{0}\right)$ is satisfied.

$$
\mathcal{L}_{v v}(\bar{v}, \bar{\lambda})[v]^{2} \geq \frac{\delta}{2}|h|_{2}^{2}>0
$$

Let us prove the converse direction. Assume, that $\left(\mathrm{SSC}_{0}\right)$ holds true but not (SSC). Then for all $\varepsilon>0$ and $\delta>0$ there exists $h_{\delta, \varepsilon} \in L^{2}(\Omega)^{n}$ such that $\left(h_{\delta, \varepsilon}\right)_{i}=0$ on $\Omega_{\varepsilon, i}, h_{\delta, \varepsilon}=u_{\delta, \varepsilon}-\bar{u}, u_{\delta, \varepsilon} \in U_{a d}$, and

$$
\mathcal{L}_{v v}(\bar{v}, \bar{\lambda})\left[\left(z_{\delta, \varepsilon}, h_{\delta, \varepsilon}\right)\right]^{2}<\delta\left|h_{\delta, \varepsilon}\right|_{2}^{2}
$$

is fulfilled with associated $z_{\delta, \varepsilon}$. Multiplying $h_{\delta, \varepsilon}$ by some positive constant, we can assume $\left|h_{\delta, \varepsilon}\right|_{2}=1$ and $h_{\delta, \varepsilon} \in T(\bar{u})$. Choosing $\delta_{k}=\varepsilon_{k}=1 / k, h_{k}:=h_{\delta_{k}, \varepsilon_{k}}$, we find

$$
\mathcal{L}_{v v}(\bar{v}, \bar{\lambda})\left[\left(z_{k}, h_{k}\right)\right]^{2}<\frac{1}{k}
$$

where $z_{k}$ is the weak solution of (3.33), hence

$$
\lim _{k} \sup \mathcal{L}_{v v}(\bar{v}, \bar{\lambda})\left[\left(z_{k}, h_{k}\right)\right]^{2} \leq 0 .
$$

Since the set $\left\{h_{k}\right\}_{k=1}^{\infty}$ is bounded in $L^{2}(\Omega)^{n}$, there exists an element $\tilde{h} \in L^{2}(\Omega)^{n}$, such that, after extracting a subsequence if necessary, the $h_{k}$ converge weakly in $L^{2}(\Omega)^{n}$ to $\tilde{h}$. The tangent cone $T(\bar{u})$ is weakly closed, therefore $\tilde{h} \in T(\bar{u})$. 
Next, we want to show $h_{k, i}(x) \rightarrow 0$ a.e. pointwise on $\Omega_{0, i}$. Let $x_{0} \in \Omega_{0, i}$ be given. Then it holds $\mid \gamma \bar{u}_{i}\left(x_{0}\right)+$ $\bar{\lambda}_{i}\left(x_{0}\right) \mid=\tau^{\prime}>0$, which implies by definition $x_{0} \in \Omega_{\tau, i}$ for all $0 \leq \tau \leq \tau^{\prime}$. Hence, there exists an index $k_{i}\left(x_{0}\right)$ such that $x_{0} \in \Omega_{\varepsilon_{k}, i}=\Omega_{1 / k, i}$ for all $k>k_{i}\left(x_{0}\right)$. By construction of $h_{k}$ we conclude $h_{k, i}\left(x_{0}\right)=0$ for all $k>k_{i}\left(x_{0}\right)$. It follows $\tilde{h}(x)=0$ on $\Omega_{0}$ almost everywhere.

We decompose $\mathcal{L}_{v v}$ and use $\left|h_{k}\right|_{2}=1$ to get

$$
\mathcal{L}_{v v}(\bar{v}, \bar{\lambda})\left[\left(z_{k}, h_{k}\right)\right]^{2}=\gamma\left|h_{k}\right|_{2}^{2}+Q\left(z_{k}\right)=\gamma+Q\left(z_{k}\right),
$$

with $Q(z)=|z|_{2}^{2}-2 b(z, z, \bar{\lambda})$. The solution mapping $h \mapsto z$ associated with (3.33) is linear and continuous from $L^{2}(\Omega)^{n}$ to $V$. Thus, we obtain $z_{k} \rightarrow \tilde{z}$ in $V$ and $z_{k} \rightarrow \tilde{z}$ in $H$, since $V$ is compactly imbedded in $H$. A wellknown result of Temam [28], Lemma II.1.5, yields $b\left(z_{k}, z_{k}, \bar{\lambda}\right) \rightarrow b(\tilde{z}, \tilde{z}, \bar{\lambda})$. We conclude $\lim _{k \rightarrow \infty} Q\left(z_{k}\right)=Q(\tilde{z})$. Passing to the limit in (3.35), we get

$$
Q(\tilde{z}) \leq \lim _{k} \sup \mathcal{L}_{v v}(\bar{v}, \bar{\lambda})\left[\left(z_{k}, h_{k}\right)\right]^{2}-\gamma \leq-\gamma<0,
$$

which proves that $\tilde{h}$ cannot vanish, remember $Q(0)=0$. Finally,

$$
\mathcal{L}_{v v}(\bar{v}, \bar{\lambda})[(\tilde{z}, \tilde{h})]^{2}=\gamma|\tilde{h}|_{2}^{2}+Q(\tilde{z}) \leq \gamma-\gamma \leq 0
$$

is obtained, which contradicts $\left(\mathrm{SSC}_{0}\right)$.

Another second-order sufficient optimality condition introduced by Casas and Mateos [8] involves the Hamiltonian of the optimal control problem. Due to the special form of our objective functional, this formulation is equivalent to $\left(\mathrm{SSC}_{0}\right)$.

Following the lines of Bonnans [4], we can prove that $\left(\mathrm{SSC}_{0}\right)$ even implies a $L^{2}$-growth condition in a $L^{2}$ neighborhood around $(\bar{y}, \bar{u})$.

Theorem 3.22. Let $\bar{v}=(\bar{y}, \bar{u})$ be admissible for the optimal control problem and suppose that $\bar{v}$ fulfills the firstorder necessary optimality condition with associated adjoint state $\bar{\lambda}$. Assume further that (SSCO) is satisfied at $\bar{v}$. Then there exist $\alpha>0$ and $\rho>0$ such that

$$
J(v) \geq J(\bar{v})+\alpha|u-\bar{u}|_{2}^{2}
$$

holds for all admissible pairs $v=(y, u)$ with $|u-\bar{u}|_{2} \leq \rho$.

Proof. Let us suppose that $\left(\mathrm{SSC}_{0}\right)$ is satisfied, whereas (3.36) does not hold. Then for all $\alpha>0$ and $\rho>0$ there exists $u_{\alpha, \rho} \in U_{a d}$ with $\left|u_{\alpha, \rho}-\bar{u}\right|_{2} \leq \rho$ and

$$
J\left(v_{\alpha, \rho}\right)<J(\bar{v})+\alpha\left|u_{\alpha, \rho}-\bar{u}\right|_{2}^{2},
$$

where $v_{\alpha, \rho}=\left(u_{\alpha, \rho}, y_{\alpha, \rho}\right)$ and $y_{\alpha, \rho}$ is the solution of (3.3) associated with $u_{\alpha, \rho}$. We choose $\alpha_{k}=\rho_{k}=1 / k$ and $u_{k}=u_{\alpha_{k}, \rho_{k}}, y_{k}=y_{\alpha_{k}, \rho_{k}}$.

By construction, it follows $u_{k} \rightarrow \bar{u}$ in $L^{2}(\Omega)^{n}$ as $k \rightarrow \infty$. Hence, we can write $u_{k}=\bar{u}+t_{k} h_{k},\left|h_{k}\right|_{2}=1$ and $t_{k} \rightarrow 0$ as $k \rightarrow \infty$. Because the set of these $h_{k}$ is bounded in $L^{2}(\Omega)^{n}$ we can extract a subsequence denoted again by $\left(h_{k}\right)$ converging weakly to $\tilde{h} \in T(\bar{u}) \subset L^{2}(\Omega)^{n}$. In the following, let $z_{k}$ be the solution of (3.33) associated with $h_{k}$.

Since $(\bar{u}, \bar{y})$ and $\left(u_{k}, y_{k}\right)$ satisfy the state equation, it holds $\mathcal{L}(\bar{v}, \bar{\lambda})=J(\bar{v})$ and $\mathcal{L}\left(v_{k}, \bar{\lambda}\right)=J\left(v_{k}\right)$. Then we obtain

$$
J\left(v_{k}\right)=\mathcal{L}\left(v_{k}, \bar{\lambda}\right)=\mathcal{L}(\bar{v}, \bar{\lambda})+t_{k} \mathcal{L}_{u}(\bar{v}, \bar{\lambda}) h_{k}+t_{k} \mathcal{L}_{y}(\bar{v}, \bar{\lambda}) z_{k}+t_{k}^{2} \mathcal{L}_{v v}\left[\left(z_{k}, h_{k}\right)^{2}\right]
$$


The first-order necessary conditions (3.15), (3.16) are fulfilled, so we find $\mathcal{L}_{y}(\bar{v}, \bar{\lambda}) z_{k}=0$ and $\mathcal{L}_{u}(\bar{v}, \bar{\lambda}) h_{k} \geq 0$. At first, we show $\tilde{h}=0$ a.e. on $\Omega_{0}$. We derive from (3.37) and (3.38)

$$
0 \leq \mathcal{L}_{u}(\bar{v}, \bar{\lambda}) h_{k}=\frac{1}{t_{k}}\left(J\left(v_{k}\right)-J(\bar{v})\right)-t_{k} \mathcal{L}_{v v}\left[\left(z_{k}, h_{k}\right)^{2}\right]<t_{k}\left\{\frac{1}{k}-\mathcal{L}_{v v}\left[\left(z_{k}, h_{k}\right)^{2}\right]\right\},
$$

which gives $\mathcal{L}_{u}(\bar{v}, \bar{\lambda}) \tilde{h}=0$ since $\mathcal{L}_{v v}\left[\left(z_{k}, h_{k}\right)^{2}\right]$ is bounded. The variational inequality

$$
\left(\gamma \bar{u}_{i}(x)+\bar{\lambda}_{i}(x)\right) h_{k, i}(x) \geq 0
$$

holds a.e. on $\Omega, i=1, \ldots, n$, so the weak limit $\tilde{h}_{i}(x)$ satisfies

$$
\left(\gamma \bar{u}_{i}(x)+\bar{\lambda}_{i}(x)\right) \tilde{h}(x) \geq 0
$$

as well. This, together with $\mathcal{L}_{u}(\bar{v}, \bar{\lambda}) \tilde{h}=0$, yields $\tilde{h}(x)=0$ on $\Omega_{0}$, cf. the definition of $\Omega_{0}$.

Finally, we show that (3.37) contradicts $\left(\mathrm{SSC}_{0}\right)$. Obviously (3.39) implies

$$
\mathcal{L}_{v v}\left[\left(z_{k}, h_{k}\right)^{2}\right]<\frac{1}{k}
$$

Arguing as in the proof of the previous Theorem 3.21, we find that $\tilde{h}$ satisfies

$$
\mathcal{L}_{v v}(\bar{v}, \bar{\lambda})[(\tilde{z}, \tilde{h})]^{2} \leq 0
$$

with $h \not \equiv 0$. Since $\tilde{h}$ is admissible as test function in $\left(\mathrm{SSC}_{0}\right)$, this shows that the positivity assumption of $\left(\mathrm{SSC}_{0}\right)$ is violated.

Remark 3.23. Observe that this theorem overcomes the two-norm discrepancy typically appearing in optimal control of semilinear equations. This is due to the very special form of the quadratic cost functional (2.1), the linear appearance of the control $u$ in the state equation, and the differentiability of the nonlinearity of the Navier-Stokes equations and the associated solution operator $G$ in weaker than $L^{\infty}$-norms.

Remark 3.24. Casas and Mateos [8] require positivity of $\mathcal{L}_{v v}$ for increments vanishing on $\Omega \backslash \Omega_{0}$ together with uniform positivity of the second derivative of the Hamiltonian with respect to the control on $\Omega \backslash \Omega_{\tau}$ for some $\tau>0$. The last property is fulfilled for our optimal control problem. The Hamiltonian is given by

$$
H(x, y, u, \lambda)=\frac{1}{2}\left|y-y_{d}(x)\right|^{2}+\frac{\gamma}{2}|u|^{2}+\lambda \cdot u .
$$

Its second derivative with respect to $u$ is

$$
\frac{\partial^{2} H}{\partial u^{2}}(x, y, u, \lambda)=\gamma,
$$

which is uniform positive on $\Omega$. Therefore, we are able to work with active sets $\Omega_{0, i}$ in $\left(\mathrm{SSC}_{0}\right)$.

In Dunn's counterexample [16], the second derivative of the Hamiltonian with respect to the control is nonnegative on $\Omega \backslash \Omega_{0}$ but indefinite on $\Omega \backslash \Omega_{\tau}$ for every $\tau>0$. Hence, the use of the active set $\Omega_{0}$ in (SSC) causes a contradiction.

\section{The instationary CASE}

In this section, we consider in a very similar way the optimal control problem (2.3)-(2.4) for the instationary Navier-Stokes equations. The similarity of arguments will permit to shorten the presentation. 


\subsection{Notations and preliminary results}

Here, we will restrict ourselve to the two-dimensional case, $n=2$, since a satisfactory theory of the instationary Navier-Stokes equations is only available for this space dimension. In the two-dimensional case, a unique weak solution of (2.4) exists that depends continuously on the given data. First, we introduce some notations and provide some results that we need later on.

To begin with, we define the solenoidal space

$$
H:=\left\{v \in L^{2}(\Omega)^{2}: \operatorname{div} v=0\right\} .
$$

Endowed with the usual $L^{2}$-scalar product, denoted by $(\cdot, \cdot)_{H}$, this space is a Hilbert space. The associated norm is denoted by $|\cdot|_{H}$. We shall work in the standard spaces of abstract functions from $[0, T]$ to a real Banach space $X, L^{p}(0, T ; X)$ and $C([0, T] ; X)$, endowed with their natural norms,

$$
\begin{aligned}
& \|y\|_{L^{p}(X)}:=\|y\|_{L^{p}(0, T ; X)}=\left(\int_{0}^{T}|y(t)|_{X}^{p} \mathrm{~d} t\right)^{1 / p}, \\
& \|y\|_{C([0, T] ; X)}:=\max _{t \in[0, T]}|y(t)|_{X}
\end{aligned}
$$

$1 \leq p<\infty$. To deal with the time derivative in (2.4), we introduce the following spaces of functions $y$ whose time derivative $y_{t}$ exists as abstract function,

$$
W^{\alpha}(0, T ; V):=\left\{y \in L^{2}(0, T ; V): y_{t} \in L^{\alpha}\left(0, T ; V^{\prime}\right)\right\},
$$

where $1 \leq \alpha<\infty$. Moreover, we write for convenience

$$
W(0, T):=W^{2}(0, T ; V) .
$$

Endowed with the norm

$$
\|y\|_{W^{\alpha}}:=\|y\|_{W^{\alpha}(0, T ; V)}=\|y\|_{L^{2}(V)}+\left\|y_{t}\right\|_{L^{\alpha}\left(V^{\prime}\right)},
$$

these spaces are Banach spaces, respectively Hilbert spaces in the case of $W(0, T)$. In the sequel, we will use for $u \in L^{p}(Q)^{2}$ the notation

$$
\|u\|_{p}:=|u|_{L^{p}(Q)^{2}} .
$$

In all what follows, $\|\cdot\|$ stands for norms of abstract functions, while $|\cdot|$ denotes norms of "stationary" spaces like $H$ and $V$.

Corollary 4.1. Let $v \in V$ and $y \in W(0, T)$ be given. It holds

$$
|v|_{4} \leq 2^{1 / 4}|v|_{H}^{1 / 2}|v|_{V}^{1 / 2}
$$

Moreover, the function $y$ is, up to changes on sets of zero measure, equivalent to a function of $C([0, T], H)$, and there is a constant $c>0$ such that

$$
\|y\|_{4}+\|y\|_{C([0, T], H)} \leq c\|y\|_{W(0, T)} .
$$

Proof. The first claim is proven in [28, Lem. III.3.3]. Note that $W(0, T)$ is continuously imbedded in $C([0, T], H)$, cf. [13]. For the $L^{4}$-claim see again in [28]. 
In view of inequality (4.1), we can state another estimate of the trilinear form $b$. In the two-dimensional case it holds

$$
|b(u, v, w)| \leq \sqrt{2}|u|_{H}^{1 / 2}|u|_{V}^{1 / 2}|v|_{V}|w|_{H}^{1 / 2}|w|_{V}^{1 / 2}
$$

for all $u, v, w \in V$. This follows directly from the estimate given in (3.1) and the previous corollary.

To specify the problem setting, we introduce a linear operator $A: L^{2}(0, T ; V) \mapsto L^{2}\left(0, T ; V^{\prime}\right)$ by

$$
\int_{0}^{T}\langle(A y)(t), v(t)\rangle_{V^{\prime}, V} \mathrm{~d} t:=\int_{0}^{T}(y(t), v(t))_{V} \mathrm{~d} t
$$

and a nonlinear operator $B: L^{2}(0, T ; V) \mapsto L^{1}\left(0, T ; V^{\prime}\right)$ by

$$
\int_{0}^{T}\langle(B(y))(t), w(t)\rangle_{V^{\prime}, V} \mathrm{~d} t:=\int_{0}^{T} b(y(t), y(t), w(t)) \mathrm{d} t
$$

where $y, v \in L^{2}(0, T ; V)$ and $w \in L^{\infty}(0, T ; V)$, respectively.

We need a bound on the admissible controls to establish a Lipschitz estimate of solutions of (2.4). Without loss of generality, we assume in the sequel that the set $U_{a d}$ is bounded in $L^{2}(Q)^{2}$, i.e. there exists a constant $\mathcal{M}>0$ such that

$$
\sup _{u \in U_{a d}}\|u\|_{2} \leq \mathcal{M}
$$

If this assumption is violated then we can introduce an artificial bound. For that purpose, let $\bar{u}$ be the optimal control and $\bar{J}=J(\bar{u})$ the corresponding value of the objective. Then $\bar{u}$ is also optimal for the same optimal control problem with changed set of admissible controls $\tilde{U}_{a d}=U_{a d} \cap\left\{u \in L^{2}(Q)^{2}:\|u\|_{2} \leq 2(\bar{J}+1) / \gamma\right\}$.

As in the stationary case, we want to derive a sufficient optimality condition that ensures local optimality of the reference control not only in $L^{\infty}(Q)^{2}$ but also in $L^{s}(Q)^{2}$, with some $s<\infty$. It remains to specify the exponent $s$.

$$
\text { (A3) }\left\{\begin{array}{c}
\text { Let } q, q^{\prime}, s \text { be real numbers such that the following statements are true. } \\
\text { (i) } q^{\prime} \leq 4 \text {. } \\
\text { (ii) The exponents } q \text { and } q^{\prime} \text { are conjugate exponents, i.e. } \frac{1}{q}+\frac{1}{q^{\prime}}=1 . \\
\text { (iii) For all } u \in L^{s}(Q)^{2} \text { it holds } \\
\|u\|_{q}^{2} \leq\|u\|_{1}\|u\|_{s} .
\end{array}\right.
$$

Here we find that the two triplets $\left(q, q^{\prime}, s\right)=(4 / 3,4,2)$ and $\left(q, q^{\prime}, s\right)=(2,2, \infty)$ fulfill this assumptions as they did in the stationary case. The assumption (i) is needed to obtain by Lemma 3.2

$$
\|y\|_{q^{\prime}} \leq(\operatorname{vol} Q)^{1 / q^{\prime}-1 / 4}\|y\|_{4}
$$

for all $y \in W(0, T)$. In the rest of this section we assume that Assumption (A3) is satisfied.

\subsection{The state equation}

We begin with the notation of weak solutions for the instationary Navier-Stokes equations (2.4)

Definition 4.2 (weak solution). Let $f \in L^{2}\left(0, T ; V^{\prime}\right)$ and $y_{0} \in H$ be given. A function $y \in L^{2}(0, T ; V)$ with $y_{t} \in L^{1}\left(0, T ; V^{\prime}\right)$ is called weak solution of $(2.4)$ if

$$
\begin{aligned}
y_{t}+\nu A y+B(y) & =f \\
y(0) & =y_{0} .
\end{aligned}
$$

Results concerning the solvability of (4.6) are standard, $c f$. [12,28] for proofs and further details. 
Theorem 4.3 (existence and uniqueness of solutions). For every $f \in L^{2}\left(0, T ; V^{\prime}\right)$ and $y_{0} \in H$, the equation (4.6) has a unique solution $y \in W(0, T)$.

Notice that the regularity $y \in W(0, T)$ is more than the regularity needed to define weak solutions. As in the stationary case, we want to work with the weakest norms of the control as possible. In the presence of a distributed control $u \in L^{2}(Q)^{2}$, the inhomogeneity $f$ is formed by

$$
\langle f(t), v(t)\rangle_{V^{\prime}, V}:=(u(t), v(t))_{q, q^{\prime}} \quad v \in L^{2}(0, T ; V), t \in[0, T],
$$

where $q$ is an exponent less or equal 2. Next we will derive some useful estimates of weak solutions. Observe, that we need $u \in L^{2}(Q)^{2}$ to prove that the solutions are of class $C([0, T], H)$, but the estimates contain $L^{q}(Q)^{2}$-norms of $u$, which are weaker since $q \leq 2$.

Lemma 4.4. For each $u \in L^{2}(Q)^{2}$ there exists a unique weak solution $y \in W(0, T)$ of $(2.4)$. It holds

$$
\|y\|_{L^{2}(V)}+\|y\|_{C([0, T], H)} \leq c_{B}\left(\left|y_{0}\right|_{H}+\|u\|_{q}\right),
$$

where $c_{B}=c_{B}(q)$ is independent of $y_{0}$ and $u$. If $y_{1}, y_{2}$ are two solutions of (2.4) associated with control functions $u_{1}, u_{2} \in U_{a d}$, repectively, then the Lipschitz estimate

$$
\left\|y_{1}-y_{2}\right\|_{L^{2}(V)}+\left\|y_{1}-y_{2}\right\|_{C([0, T], H)} \leq c_{L}\left\|u_{1}-u_{2}\right\|_{q}
$$

is satisfied with some constant $c_{L}>0$.

Proof. Existence and regularity follow from Theorem 4.3. Let $y$ be the unique weak solution of (2.4) defined by (4.6). We test (4.6) by $y$. Then the nonlinear term vanishes due to $b(y(t), y(t), y(t))=0$ for almost all $t \in[0, T]$. We get the following differential equation:

$$
\frac{1}{2} \frac{\mathrm{d}}{\mathrm{d} t}|y(t)|_{H}^{2}+\nu|y(t)|_{V}^{2}=(u(t), y(t))_{q, q^{\prime}} \quad \text { a.e. on }[0, T]
$$

Integration from 0 to $t \in[0, T]$ yields

$$
\frac{1}{2}|y(t)|_{H}^{2}-\frac{1}{2}\left|y_{0}\right|_{H}^{2}+\nu \int_{0}^{t}|y(s)|_{V}^{2} \mathrm{~d} s=\int_{0}^{t}(u(s), y(s))_{q, q^{\prime}} \mathrm{d} s .
$$

Using Hölders inequality, the inequalities (4.1), (4.5), and Young's inequality, we derive

$$
\begin{aligned}
\int_{0}^{t}(u, y)_{q, q^{\prime}} \mathrm{d} s & \leq \int_{0}^{t}|u(s)|_{q}|y(s)|_{q^{\prime}} \mathrm{d} s \leq\left(\int_{0}^{t}|u(s)|_{q}^{q} \mathrm{~d} s\right)^{1 / q}\left(\int_{0}^{t}|y(s)|_{q^{\prime}}^{q^{\prime}} \mathrm{d} s\right)^{1 / q^{\prime}} \\
& \leq c_{a}\|u\|_{q}\left(\int_{0}^{t}|y(s)|_{H}^{q^{\prime} / 2}|y(s)|_{V}^{q^{\prime} / 2} \mathrm{~d} s\right)^{1 / q^{\prime}} \\
& \leq c_{a}\|u\|_{q}\|y\|_{C([0, T], H)}^{1 / 2}\|y\|_{L^{q^{\prime} / 2}(0, t ; V)}^{1 / 2}
\end{aligned}
$$

where $c_{a}=2^{1 / 4}(\operatorname{vol} Q)^{\mu}$ and $\mu=1 / q^{\prime}-1 / 4$ are given by (4.1) and (4.5). Notice, that $q^{\prime} \leq 4$ implies $q^{\prime} / 2 \leq 2$, hence we can apply Lemma 3.2 with respect to the time interval $[0, t]$ to proceed

$$
\begin{aligned}
& \leq c_{a} T^{2 \mu}\|u\|_{q}\|y\|_{C([0, T], H)}^{1 / 2}\|y\|_{L^{2}(0, t ; V)}^{1 / 2} \\
& \leq c_{b}\|u\|_{q}^{2}+\frac{1}{4}\|y\|_{C([0, T], H)}^{2}+\frac{\nu}{2}\|y\|_{L^{2}(0, t ; V)}^{2},
\end{aligned}
$$


where $c_{b}=\frac{1}{2}(\operatorname{vol} Q)^{2 \mu} T^{4 \mu} \nu^{-1 / 2}$. Putting (4.9) and (4.10) together, we find that

$$
\frac{1}{2}|y(t)|_{H}^{2}+\frac{\nu}{2}\|y\|_{L^{2}(0, t ; V)}^{2} \leq \frac{1}{2}\left|y_{0}\right|_{H}^{2}+c_{b}\|u\|_{q}^{2}+\frac{1}{4}\|y\|_{C([0, T], H)}^{2}
$$

holds for almost all $t \in[0, T]$. Here, the $C([0, T], H)$-norm of $y$ appears on the right-hand side to bound $|y(t)|_{H}$. Since $y \in C([0, T], H)$ is given by Theorem 4.3, this inequality makes sense. Taking the maximum for $t \in[0, T]$ on the left-hand side we get

$$
\frac{1}{4}\|y\|_{C([0, T], H)}^{2} \leq \frac{1}{2}\left|y_{0}\right|_{H}^{2}+c_{b}\|u\|_{q}^{2} .
$$

The $L^{2}(0, T ; V)$-estimate of $y$ follows immediately,

$$
\|y\|_{L^{2}(V)}^{2} \leq \frac{2}{\nu}\left|y_{0}\right|_{H}^{2}+\frac{4 c_{b}}{\nu}\|u\|_{q}^{2} \leq \frac{2}{\nu}\left|y_{0}\right|_{H}^{2}+\frac{2}{\nu^{3 / 2}} \mathcal{M}^{2}=: \mathcal{K} .
$$

In this way, we have derived a uniform bound on $\|y\|_{L^{2}(V)}$ for all states $y$ associated with admissible controls. It remains to prove the Lipschitz-estimate. Let $y_{1}, y_{2}$ be two solutions of (2.4) associated with the control functions $u_{1}, u_{2}$. Denote by $y$ and $u$ the difference of them, $y=y_{1}-y_{2}$ and $u=u_{1}-u_{2}$. We substract the corresponding variational equalities, test with $v=y$, and integrate over $[0, t]$. This yields

$$
\frac{1}{2}|y(t)|_{H}^{2}+\nu \int_{0}^{t}|y(s)|_{V}^{2} \mathrm{~d} s=\int_{0}^{t}(u(s), y(s))_{q, q^{\prime}} \mathrm{d} s-\int_{0}^{t} b\left(y(s), y_{2}(s), y(s)\right) \mathrm{d} s,
$$

since $y(0)=y_{1}(0)-y_{2}(0) \equiv 0$. For the treatment of the nonlinear terms we refer to equation (3.7). Analogously as above, we conclude

$$
\int_{0}^{t}(u(s), y(s))_{q, q^{\prime}} \mathrm{d} s \leq c_{u}\|u\|_{q}^{2}+\frac{1}{\mathcal{N}}\|y\|_{C([0, T], H)}^{2}+\frac{\nu}{2}\|y\|_{L^{2}(0, t ; V)}^{2},
$$

with $c_{u}=\frac{1}{4}(\operatorname{vol} Q)^{2 \mu} T^{4 \mu} \mathcal{N}^{1 / 2} \nu^{-1 / 2}$ and a constant $\mathcal{N}>0$ to be specified later. The nonlinear term is estimated by $(4.3)$,

$$
\begin{aligned}
\left|\int_{0}^{t} b\left(y(s), y_{2}(s), y(s)\right) \mathrm{d} s\right| & \leq \sqrt{2} \int_{0}^{t}|y(s)|_{H}|y(s)|_{V}\left|y_{2}(s)\right|_{V} \mathrm{~d} s \\
& \leq \frac{\nu}{4} \int_{0}^{t}|y(s)|_{V}^{2} \mathrm{~d} s+\frac{2}{\nu} \int_{0}^{t}|y(s)|_{H}^{2}\left|y_{2}(s)\right|_{V}^{2} \mathrm{~d} s .
\end{aligned}
$$

Inserting these estimates in (4.12), we obtain

$$
\frac{1}{2}|y(t)|_{H}^{2}+\frac{\nu}{4}\|y\|_{L^{2}(0, t ; V)}^{2} \leq c_{u}\|u\|_{q}^{2}+\frac{1}{\mathcal{N}}\|y\|_{C([0, T], H)}^{2}+\frac{2}{\nu} \int_{0}^{t}|y(s)|_{H}^{2}\left|y_{2}(s)\right|_{V}^{2} \mathrm{~d} s .
$$

Since $y_{2} \in L^{2}(0, T ; V)$, the norm square $\left|y_{2}(\cdot)\right|_{V}^{2}$ is integrable and Gronwall's lemma applies to get

$$
|y(t)|_{H}^{2} \leq \exp \left(\frac{4}{\nu}\left\|y_{2}\right\|_{L^{2}(V)}^{2}\right)\left(c_{u}\|u\|_{q}^{2}+\frac{2}{\mathcal{N}}\|y\|_{C([0, T], H)}^{2}\right) .
$$

We choose $\mathcal{N}:=8 \exp \left(\frac{4}{\nu} \mathcal{K}\right)$, where $\mathcal{K}$ is given by (4.11). The uniform bound derived also in equation (4.11) yields that the following inequality holds for all $t \in[0, T]$ :

$$
\frac{1}{2}|y(t)|_{H}^{2} \leq \frac{1}{4}\|y\|_{C([0, T], H)}^{2}+c\|u\|_{q}^{2}
$$


With the same arguments as above, we conclude

$$
\|y\|_{C([0, T], H)}+\|y\|_{L^{2}(V)} \leq c\|u\|_{q}
$$

and the Lipschitz dependence of the states on the controls is proven.

To establish optimality conditions, we will also need estimates of solutions of linearized equations. Therefore, we introduce the derivative $B^{\prime}\left(y_{l}\right)$ of the nonlinear operator $B$ which is given by

$$
\int_{0}^{T}\left\langle B^{\prime}\left(y_{l}(t)\right) y(t), w(t)\right\rangle_{V^{\prime}, V} \mathrm{~d} t:=\int_{0}^{T}\left\{b\left(y_{l}(t), y(t), w(t)\right)+b\left(y(t), y_{l}(t), w(t)\right)\right\} \mathrm{d} t .
$$

In view of (4.3), it can be shown that $B^{\prime}\left(y_{l}\right) \in \mathcal{L}\left(L^{2}(0, T ; V), L^{4 / 3}\left(0, T ; V^{\prime}\right)\right)$ for $y_{l} \in W(0, T)$.

Lemma 4.5. Let $y_{l} \in W(0, T)$ be the state associated with a control $u_{l} \in U_{a d}$. Then, for all $u \in L^{2}(Q)^{2}$, there exists a unique weak solution $y \in W(0, T)$ of the linearized equation

$$
\begin{aligned}
y_{t}+\nu A y+B^{\prime}\left(y_{l}\right) y & =u, \\
y(0) & =0 .
\end{aligned}
$$

It satisfies the estimate

$$
\|y\|_{C([0, T], H)}+\|y\|_{L^{2}(V)} \leq c_{l}\|u\|_{q} .
$$

Proof. For the proof of existence we refer to [20]. A similar result was proven in [7] for the three-dimensional case. The estimate (4.14) can be shown as in the previous lemma. The uniqueness of solutions is a consequence of the linearity of the equation and the continuity estimate (4.14).

\subsection{First order necessary optimality conditions}

Now we return to our optimal control problem. Before stating the second-order sufficient optimality condition, we briefly recall the necessary conditions for local optimality. For the proofs and further discussion see $[1,7,19,21]$ and the references cited therein.

Definition 4.6 (locally optimal control). A control $\bar{u} \in U_{a d}$ is said to be locally optimal in $L^{2}(Q)^{2}$, if there exists a constant $\rho>0$ such that

$$
J(\bar{y}, \bar{u}) \leq J\left(y_{h}, u_{h}\right)
$$

holds for all $u_{h} \in U_{a d}$ with $\left\|\bar{u}-u_{h}\right\|_{2} \leq \rho$. Here, $\bar{y}$ and $y_{h}$ denote the states associated with $\bar{u}$ and $u_{h}$, respectively.

In the following, we denote by $B^{\prime}(\bar{y})^{*}$ the adjoint of $B^{\prime}(\bar{y})$. For $\bar{y} \in W(0, T)$, it is a continuous linear operator from $L^{2}(0, T ; V)$ to $L^{4 / 3}\left(0, T ; V^{\prime}\right)$.

Theorem 4.7 (necessary condition). Let $\bar{u}$ be a locally optimal control with associated state $\bar{y}=y(\bar{u})$. Then there exists a unique solution $\bar{\lambda} \in W^{4 / 3}(0, T ; V)$ of the adjoint equation

$$
\begin{aligned}
-\bar{\lambda}_{t}+\nu A \bar{\lambda}+B^{\prime}(\bar{y})^{*} \bar{\lambda} & =\bar{y}-y_{Q} \\
\bar{\lambda}(T) & =\bar{y}(T)-y_{T} .
\end{aligned}
$$

Moreover, the variational inequality

$$
(\gamma \bar{u}+\bar{\lambda}, u-\bar{u})_{L^{2}(Q)^{2}} \geq 0 \quad \forall u \in U_{a d}
$$

is satisfied. 
Proof. A proof can be found in [19,20]. It can be carried out along the lines of the proof of Theorem 3.10 for the stationary case. First, one can show the Fréchet-differentiablity of the solution operator of the instationary equation. The adjoint system and the variational inequality is then derived by the method of transposition. The regularity of $\bar{\lambda}$ is proven in [22].

Next, we state an estimate of the norm of the adjoint state, see [21] for the details.

Corollary 4.8. Let $\lambda \in W^{4 / 3}(0, T ; V)$ be the weak solution of (4.15), where $y \in W(0, T)$ is a state associated with an admissible control $u \in U_{\text {ad }}$. Then it holds

$$
\|\lambda\|_{L^{2}(V)}^{2} \leq c\left(\left|y(T)-y_{T}\right|_{L^{2}(\Omega)}^{2}+\left\|y-y_{Q}\right\|_{2}^{2}\right) .
$$

Let us introduce the Lagrange function $\mathcal{L}: W(0, T) \times L^{2}(Q)^{2} \times W^{4 / 3}(0, T ; V)$ of the instationary optimal control problem by

$$
\mathcal{L}(y, u, \lambda)=J(u, y)+\int_{0}^{T}\left\{\left\langle y_{t}, \lambda\right\rangle_{V^{\prime}, V}-\nu(y, \lambda)_{V}-b(y, y, \lambda)+(u, \lambda)\right\} \mathrm{d} t .
$$

One can easily verify that the necessary optimality conditions given in Theorem 4.7 are equivalent to

$$
\mathcal{L}_{u}(\bar{y}, \bar{u}, \bar{\lambda})(u-\bar{u}) \geq 0 \quad \forall u \in U_{a d},
$$

and

$$
\mathcal{L}_{y}(\bar{y}, \bar{u}, \bar{\lambda}) h=0 \quad \forall h \in W(0, T) \text { with } h(0)=0 .
$$

As in the stationary case, it can be proven that $\mathcal{L}$ is twice Fréchet-differentiable with respect to $y$ and $u$, confer Lemma 3.12. Here we derive an estimate of the norm of $\mathcal{L}_{y y}$.

In the analysis of the second-order condition estimations of the time derivative $y_{t}$ of a state $y \in W(0, T)$ are not needed. Therefore, we introduce a space $\tilde{W}$ by

$$
\tilde{W}=L^{2}(0, T ; V) \cap C([0, T], H) .
$$

equipped with the norm

$$
\|y\|_{\tilde{W}}^{2}:=\|y\|_{L^{2}(V)}^{2}+\|y\|_{C([0, T], H)}^{2} .
$$

Lemma 4.9. The second derivative of the Lagrangian $\mathcal{L}$ at $y \in W(0, T)$ with associated adjoint state $\lambda$ in the direction $z_{1}, z_{2} \in W(0, T)$ satisfies the estimate

$$
\left|\mathcal{L}_{y y}(y, u, \lambda)\left[z_{1}, z_{2}\right]\right| \leq c_{\mathcal{L}}\left\|z_{1}\right\|_{\tilde{W}}\left\|z_{2}\right\|_{\tilde{W}}
$$

for all $z_{1}, z_{2} \in W(0, T)$.

Proof. The second derivative of $\mathcal{L}$ is given by

$$
\mathcal{L}_{y y}(y, u, \lambda)\left[z_{1}, z_{2}\right]=\left(z_{1}(T), z_{2}(T)\right)_{2}+\int_{0}^{T}\left(z_{1}, z_{2}\right)_{2}-b\left(z_{1}, z_{2}, \lambda\right)-b\left(z_{2}, z_{1}, \lambda\right) \mathrm{d} t
$$


The nonlinear terms are estimated by (4.3),

$$
\begin{aligned}
\left|\int_{0}^{T} b\left(z_{1}, z_{2}, \lambda\right) \mathrm{d} t\right| & \leq c \int_{0}^{T}\left|z_{1}(t)\right|_{H}^{1 / 2}\left|z_{1}(t)\right|_{V}^{1 / 2}\left|z_{2}(t)\right|_{H}^{1 / 2}\left|z_{2}(t)\right|_{V}^{1 / 2}|\lambda(t)|_{V} \mathrm{~d} t \\
& \leq c\left\|z_{1}\right\|_{C([0, T], H)}^{1 / 2}\left\|z_{2}\right\|_{C([0, T], H)}^{1 / 2} \int_{0}^{T}\left|z_{1}(t)\right|_{V}^{1 / 2}\left|z_{2}(t)\right|_{V}^{1 / 2}|\lambda(t)|_{V} \mathrm{~d} t \\
& \leq c\left\|z_{1}\right\|_{C([0, T], H)}^{1 / 2}\left\|z_{2}\right\|_{C([0, T], H)}^{1 / 2}\left\|z_{1}\right\|_{L^{2}(V)}^{1 / 2}\left\|z_{2}\right\|_{L^{2}(V)}^{1 / 2}\|\lambda\|_{L^{2}(V)} \\
& \leq c\left\|z_{1}\right\|_{\tilde{W}}\left\|z_{2}\right\|_{\tilde{W}}\|\lambda\|_{L^{2}(V)} .
\end{aligned}
$$

Corollary 4.8 together with Lemma 4.4 and the boundedness of the controls (4.4) yields a uniform bound on all adjoint states, $\|\lambda\|_{L^{2}(V)} \leq C$, independently of $y$ and $u$. Now the claim follows immediately.

\subsection{Second-order sufficient optimality condition}

In what follows we fix $\bar{v}:=(\bar{y}, \bar{u})$ to be an admissible reference pair. We suppose that $\bar{v}$ satisfies the first-order necessary optimality conditions.

Definition 4.10 (strongly active sets). Let $\varepsilon>0$ and $i \in\{1,2\}$ be given. Define sets $Q_{\varepsilon, i} \subseteq Q=\Omega \times[0, T]$ by

$$
Q_{\varepsilon, i}=\left\{(x, t) \in Q:\left|\gamma \bar{u}_{i}(x, t)+\bar{\lambda}_{i}(x, t)\right|>\varepsilon\right\} .
$$

For $u \in L^{p}(Q)^{2}$ and $1 \leq p<\infty$ we define the $L^{p}$-norm with respect to the sets of strongly active control constraints

$$
\|u\|_{L^{p}, Q_{\varepsilon}}:=\left(\sum_{i=1}^{2}\left\|u_{i}\right\|_{L^{p}\left(Q_{\varepsilon, i}\right)}^{p}\right)^{1 / p} .
$$

As in the previous section, we can show the following conclusion, $c f$. Corollary 3.14 .

Corollary 4.11. For all $u \in U_{\text {ad }}$ it holds

$$
\sum_{i=1}^{2} \int_{0}^{T} \int_{Q_{\varepsilon, i}}\left(\gamma \bar{u}_{i}(x, t)+\bar{\lambda}_{i}(x, t)\right)\left(u_{i}(x, t)-\bar{u}_{i}(x, t)\right) \mathrm{d} x \mathrm{~d} t \geq \varepsilon\|u-\bar{u}\|_{L^{1}\left(Q_{\varepsilon}\right)} .
$$

We assume that the reference pair $\bar{v}=(\bar{y}, \bar{u})$ satisfies the following coercivity assumption on $\mathcal{L}^{\prime \prime}(\bar{v}, \bar{\lambda})$, in the sequel called second-order sufficient condition:

(SSC)

$$
\left\{\begin{array}{l}
\text { There exist } \varepsilon>0 \text { and } \delta>0 \text { such that } \\
\qquad \mathcal{L}_{v v}(\bar{v}, \bar{\lambda})[(z, h)]^{2} \geq \delta\|h\|_{q}^{2} \\
\text { holds for all pairs }(z, h) \in W(0, T) \times L^{2}(Q)^{2} \text { with } \\
\qquad \begin{array}{r}
h=u-\bar{u}, u \in U_{a d}, h_{i}=0 \text { on } Q_{\varepsilon, i} \text { for } i=1,2, \\
\text { and } z \in W(0, T) \text { being the weak solution of the linearized equation } \\
z_{t}+A z+B^{\prime}(\bar{y}) z=h \\
z(0)=0 .
\end{array}
\end{array}\right.
$$

Now, we collected all tools to prove that (SSC) is sufficient for local optimality of $(\bar{y}, \bar{u})$, provided the first-order necessary conditions are fulfilled. The proof in the instationary case follows exactly the lines of the proof in the stationary case, $c f$. Theorem 3.17. So we only state the associated results without proof. 
Theorem 4.12. Let $\bar{v}=(\bar{y}, \bar{u})$ be admissible for the optimal control problem and suppose that $\bar{v}$ fulfills the first-order necessary optimality condition with associated adjoint state $\bar{\lambda}$. Assume further that (SSC) is satisfied at $\bar{v}$. Then there exist $\alpha>0$ and $\rho>0$ such that

$$
J(v) \geq J(\bar{v})+\alpha\|u-\bar{u}\|_{q}^{2}
$$

holds for all admissible pairs $v=(y, u)$ with $\|u-\bar{u}\|_{s} \leq \rho$, where the exponents $s$ and $q$ are chosen according to Assumption (A3).

Remark 4.13. As in the stationary case, cf. Section 3.4.2, one can establish an equivalent sufficient condition $\left(\mathrm{SSC}_{0}\right)$, which ensures together with first-order necessary optimality conditions local optimality of a reference control without any two-norm discrepancy. However, here one needs extra regularity requirements for the data.

Acknowledgements. The authors are very grateful to E. Casas for his comments that led us to the remarks in Section $\mu 3.4 .2$.

\section{REFERENCES}

[1] F. Abergel and R. Temam, On some control problems in fluid mechanics. Theoret. Comput. Fluid Dynam. 1 (1990) $303-325$.

[2] R.A. Adams, Sobolev spaces. Academic Press, San Diego (1978).

[3] N. Arada, J.-P. Raymond and F. Tröltzsch, On an augmented Lagrangian SQP method for a class of optimal control problems in Banach spaces. Comput. Optim. Appl. 22 (2002) 369-398.

[4] J.F. Bonnans, Second-order analysis for control constrained optimal control problems of semilinear elliptic equations. Appl. Math. Optim. 38 (1998) 303-325.

[5] J.F. Bonnans and H. Zidani, Optimal control problems with partially polyhedric constraints. SIAM J. Control Optim. $\mathbf{3 7}$ (1999) 1726-1741.

[6] H. Brezis, Analyse fonctionelle. Masson, Paris (1983).

[7] E. Casas, An optimal control problem governed by the evolution Navier-Stokes equations, in Optimal control of viscous flows. Frontiers in applied mathematics, S.S. Sritharan Ed., SIAM, Philadelphia (1993).

[8] E. Casas and M. Mateos, Second order optimality conditions for semilinear elliptic control problems with finitely many state constraints. SIAM J. Control Optim. 40 (2002) 1431-1454.

[9] E. Casas and M. Mateos, Uniform convergence of the FEM. Applications to state constrained control problems. Comp. Appl. Math. 21 (2002) 67-100.

[10] E. Casas, F. Tröltzsch and A. Unger, Second-order sufficient optimality conditions for a nonlinear elliptic control problem. $J$. Anal. Appl. 15 (1996) 687-707.

[11] E. Casas, F. Tröltzsch and A. Unger, Second-order sufficient optimality conditions for some state-constrained control problems of semilinear elliptic equations. SIAM J. Control Optim. 38 (2000) 1369-1391.

[12] P. Constantin and C. Foias, Navier-Stokes equations. The University of Chicago Press, Chicago (1988).

[13] R. Dautray and J.L. Lions, Evolution problems I, Mathematical analysis and numerical methods for science and technology $\mathbf{5}$. Springer, Berlin (1992).

[14] M. Desai and K. Ito, Optimal controls of Navier-Stokes equations. SIAM J. Control Optim. 32 (1994) 1428-1446.

[15] A.L. Dontchev, W.W. Hager, A.B. Poore and B. Yang, Optimality, stability, and convergence in optimal control. Appl. Math. Optim. 31 (1995) 297-326.

[16] J.C. Dunn, On second-order sufficient conditions for structured nonlinear programs in infinite-dimensional function spaces, in Mathematical programming with data perturbations, A. Fiacco Ed., Marcel Dekker (1998) 83-107.

[17] H.O. Fattorini and S. Sritharan, Necessary and sufficient for optimal controls in viscous flow problems. Proc. Roy. Soc. Edinburgh 124 (1994) 211-251.

[18] M.D. Gunzburger Ed., Flow control. Springer, New York (1995).

[19] M.D. Gunzburger and S. Manservisi, The velocity tracking problem for Navier-Stokes flows with bounded distributed controls. SIAM J. Control Optim. 37 (1999) 1913-1945.

[20] M.D. Gunzburger and S. Manservisi, Analysis and approximation of the velocity tracking problem for Navier-Stokes flows with distributed control. SIAM J. Numer. Anal. 37 (2000) 1481-1512.

[21] M. Hinze, Optimal and instantaneous control of the instationary Navier-Stokes equations. Habilitation, TU Berlin (2002). 
[22] M. Hinze and K. Kunisch, Second-order methods for optimal control of time-dependent fluid flow. SIAM J. Control Optim. 40 (2001) 925-946.

[23] H. Maurer and J. Zowe, First- and second-order conditions in infinite-dimensional programming problems. Math. Programming 16 (1979) 98-110.

[24] H.D. Mittelmann and F. Tröltzsch, Sufficient optimality in a parabolic control problem, in Trends in Industrial and Applied Mathematics, A.H. Siddiqi and M. Kocvara Ed., Dordrecht, Kluwer (2002) 305-316.

[25] J.-P. Raymond and F. Tröltzsch, Second order sufficient optimality conditions for nonlinear parabolic control problems with state constraints. Discrete Contin. Dynam. Syst. 6 (2000) 431-450.

[26] T. Roubíček and F. Tröltzsch, Lipschitz stability of optimal controls for the steady-state Navier-Stokes equations. Control Cybernet. 32 (2002) 683-705.

[27] S. Sritharan, Dynamic programming of the Navier-Stokes equations. Syst. Control Lett. 16 (1991) 299-307.

[28] R. Temam, Navier-Stokes equations. North Holland, Amsterdam (1979).

[29] F. Tröltzsch, Lipschitz stability of solutions of linear-quadratic parabolic control problems with respect to perturbations. Dyn. Contin. Discrete Impulsive Syst. 7 (2000) 289-306. 